Prepared for the U.S. Department of Energy, Office of Civilian Radioactive Waste Management, Office of Repository Development under Interagency Agreements DE-AI28-02RW12167 and DE-AI28-07RW12405

\title{
Meteoric Precipitation at Yucca Mountain, Nevada: Chemical and Stable Isotope Analyses, 2006-09
}

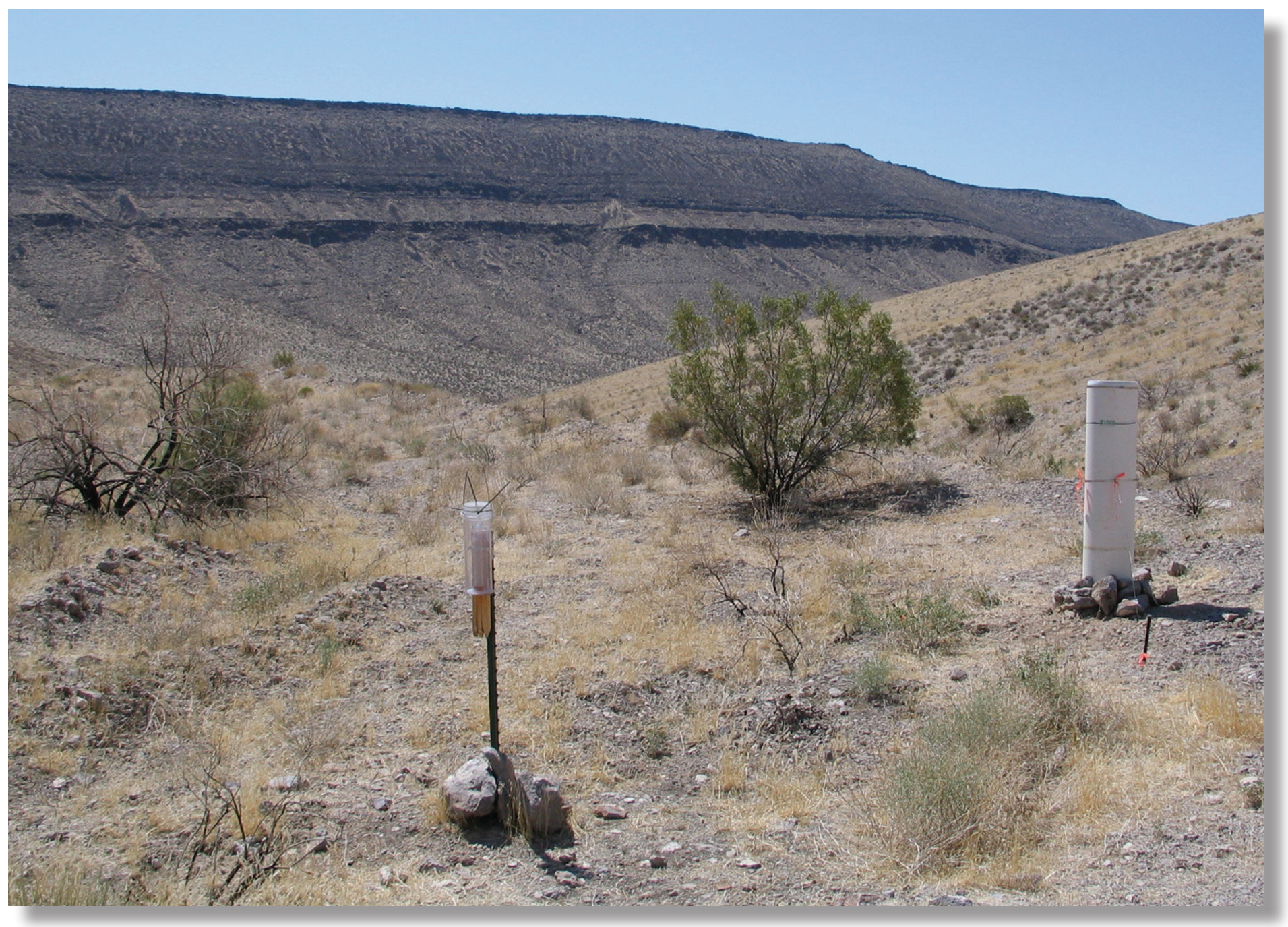

Scientific Investigations Report 2011-5140 
Cover: Precipitation collectors for isotope and chemistry samples in Solitario Canyon. Northsouth trending ridge line of Yucca Mountain can be seen against the sky (Photograph by Richard J. Moscati, U.S. Geological Survey). 


\section{Meteoric Precipitation at Yucca Mountain, Nevada: Chemical and Stable Isotope Analyses, 2006-09}

By Richard J. Moscati and Kevin M. Scofield

Prepared for the U.S. Department of Energy,

Office of Civilian Radioactive Waste Management, Office of Repository Development under Interagency Agreements DE-Al28-02RW12167 and DE-AI28-07RW12405

Scientific Investigations Report 2011-5140 


\title{
U.S. Department of the Interior \\ KEN SALAZAR, Secretary \\ U.S. Geological Survey \\ Marcia K. McNutt, Director
}

\author{
U.S. Geological Survey, Reston, Virginia: 2011
}

For more information on the USGS - the Federal source for science about the Earth, its natural and living resources, natural hazards, and the environment, visit http://www.usgs.gov or call 1-888-ASK-USGS.

For an overview of USGS information products, including maps, imagery, and publications, visit http://www.usgs.gov/pubprod

To order this and other USGS information products, visit http://store.usgs.gov

Any use of trade, product, or firm names is for descriptive purposes only and does not imply endorsement by the U.S. Government.

Although this report is in the public domain, permission must be secured from the individual copyright owners to reproduce any copyrighted materials contained within this report.

Permission must be secured from the individual

copyright owners to reproduce any copyrighted material contained within this report.

Suggested citation:

Moscati, R.J., and Scofield, K.M., 2011, Meteoric precipitation at Yucca Mountain, Nevada:

Chemical and stable isotope analyses, 2006-09: U.S. Geological Survey Scientific

Investigations Report 2011-5140, 16 p. 


\section{Contents}

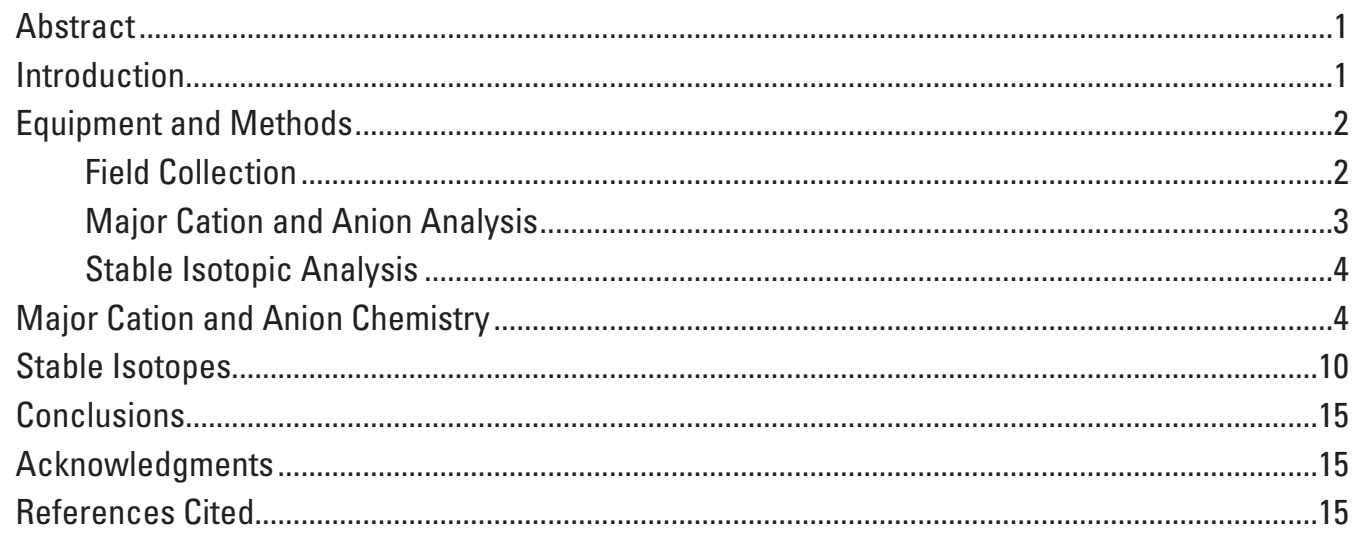

\section{Figures}

1. Shaded relief map of Yucca Mountain, Nevada, showing precipitation collection sites and the Exploratory Studies Facility.

2. Photograph of precipitation collectors for isotope and chemistry samples at Yucca Mountain, Nevada.

3. Relative concentrations on piper diagram, in percent milliequivalents per liter, of the major ions in precipitation samples from monitoring stations at Yucca Mountain, Nevada, December 2007 to January 2009

4. Graph showing delta deuterium plotted against delta oxygen-18, in per mil relative to Vienna Standard Mean Ocean Water, for 2006-2009 precipitation samples from monitoring stations at Yucca Mountain, Nevada.

5. Graph showing delta deuterium plotted against delta oxygen-18, in per mil relative to Vienna Standard Mean Ocean Water, for 2006-2009 samples of winter and summer precipitation from monitoring stations at Yucca Mountain, Nevada

\section{Tables}

1. Location of precipitation collection stations at Yucca Mountain, Nevada.........................3

2. Major cation and anion concentrations for precipitation samples at Yucca Mountain, Nevada, December 2007 to January 2009...

3. Delta oxygen-18 $\left(\delta^{18} 0\right)$ and delta deuterium $(\delta \mathrm{D})$ values, and amounts, of precipitation samples at Yucca Mountain, Nevada, February 2006 to January 2009 


\section{Conversion Factors and Abbreviations}

\begin{tabular}{lll}
\hline \multicolumn{1}{c}{ Multiply } & By & \multicolumn{1}{c}{ To obtain } \\
\hline millimeter $(\mathrm{mm})$ & Length & \\
centimeter $(\mathrm{cm})$ & 0.03937 & inch (in) \\
meter $(\mathrm{m})$ & 0.3937 & inch (in) \\
kilometer $(\mathrm{km})$ & 3.2808 & feet (ft) \\
& 0.6214 & mile (mi) \\
\hline microliter $(\mu \mathrm{L})$ & Volume & \\
milliliter $(\mathrm{mL})$ & $3.382 \times 10^{-5}$ & ounce, fluid (fl. oz) \\
\hline
\end{tabular}

Stable isotope compositions are reported as delta $(\delta)$ values in units of parts per thousand (denoted as per mil or \%) relative to a standard of known composition (Craig, 1961): Pee Dee Belemnite (PDB) for carbon (C), and Vienna Standard Mean Ocean Water (VSMOW) for oxygen (0).

Mil is a unit of length equal to one thousandth $\left(10^{-3}\right)$ of an inch ( 0.0254 millimeter), used, for example, to specify the diameter of wire or the thickness of materials sold in sheets.

Chemical concentrations are given in metric units. Chemical concentration is given in milligrams per liter (mg/L). Milligrams per liter (equivalent to parts per million) is a unit expressing the concentration of chemical constituents in solution as weight (milligrams) of solute per unit volume (liter) of water. Specific conductance (or conductivity) is given in microsiemens per centimeter at $25^{\circ} \mathrm{C}(\mu \mathrm{S} / \mathrm{cm})$.

Vertical coordinate information is referenced to the North American Datum of 1927.

Temperature in degrees Celsius $\left({ }^{\circ} \mathrm{C}\right)$ may be converted to degrees Fahrenheit $\left({ }^{\circ} \mathrm{F}\right)$ as follows:

$$
{ }^{\circ} \mathrm{F}=\left(1.8 \mathrm{x}^{\circ} \mathrm{C}\right)+32
$$




\title{
Meteoric Precipitation at Yucca Mountain, Nevada: Chemical and Stable Isotope Analyses, 2006-09
}

\author{
By Richard J. Moscati and Kevin M. Scofield
}

\section{Abstract}

Meteoric precipitation samples collected in 2006-09 at Yucca Mountain, Nevada, were analyzed for chemistry and stable isotope composition. Precipitation is the major source of infiltration to the unsaturated zone and of recharge to the saturated zone at Yucca Mountain.

On February 28, 2005, seepage of water was observed about 40 to $80 \mathrm{~m}$ below the ground surface within the Tiva Canyon Tuff in the South Ramp of the Exploratory Studies Facility (ESF) tunnel within Yucca Mountain. This seepage was preceded by a 5 -month period of above-average precipitation. Chemical and isotopic analysis of this seepage could not be used to estimate travel time, extent of water-rock interaction, and (or) evaporative water loss during percolation through the unsaturated zone due to the lack of corresponding chemical and isotopic analyses of precipitation inputs to the infiltration events that produced the seepage.

In February 2006, collection of precipitation samples for chemical (major ions), delta oxygen-18 $\left(\delta^{18} \mathrm{O}\right)$, and delta deuterium $(\delta \mathrm{D})$ analyses began at seven meteorological monitoring stations to provide baseline isotopic and chemical analyses. The sampling stations range in elevation from 1,131 to 1,562 meters. Each site has two collectors - one for chemical analysis and the other for isotopic analysis of precipitation. The collectors were sampled and emptied after each precipitation event.

In 2006-09, 36 distinct precipitation events, with an average 3- to 4-day duration and an average 9.9 millimeters of accumulation, have been analyzed. The chemical composition of these samples of Yucca Mountain precipitation is relatively dilute but contains measurable and variable concentrations of $\mathrm{Na}^{+}, \mathrm{Ca}^{2+}, \mathrm{NH}_{4}^{+}, \mathrm{NO}_{3}^{-}$, and $\mathrm{SO}_{4}^{2-}$. Dust transported by typical winter storms and generated from soil carbonates is the main contributor to this precipitation chemistry. Elevated nitrate and ammonium concentrations may be linked to agriculture in the nearby Amargosa River valley to the south and west of Yucca Mountain.

Cumulatively, $\delta^{18} \mathrm{O}$ values range from 3.0 to -20.4 per mil $(\%)$ and $\delta \mathrm{D}$ values range from 10 to $-147 \%$. Winterseason precipitation commonly has isotopically lighter compositions. The cumulative $\delta^{18} \mathrm{O}$ plotted against $\delta \mathrm{D}$ shows that precipitation samples define a line with slope of 6.4, less than the 8 of the Global Meteoric Water Line. This difference in slope, typical of arid environments, is chiefly the result of evaporation of falling raindrops due to warmer air temperatures.

\section{Introduction}

Meteoric precipitation is the major source of infiltration to the unsaturated zone and of recharge of water to the saturated zone at Yucca Mountain, Nevada, a location studied for a formerly proposed nuclear waste repository (Levich and Stuckless, 2007). On February 28, 2005, seepage of water was observed in the form of wet spots on the crown, ribs, and invert of the Exploratory Studies Facility (ESF) tunnel, between stations 75+62 and 77+52 (Oliver and Whelan, 2006). (ESF locations are defined by stations at 100 -meter (m) intervals; for example, station $75+62$ is 7,562 $\mathrm{m}$ from the reference point near the north portal of the ESF). Stations $75+62$ and $77+52$ are 315 and $125 \mathrm{~m}$, respectively, from the ESF South Portal (fig. 1). The seepage area was about 40 to $80 \mathrm{~m}$ below the ground surface within the approximately 12.7 million-year-old Miocene-age (Sawyer and others, 1994) Tiva Canyon Tuff middle nonlithophysal and lower nonlithophysal units (Buesch and others, 1996). The observed seepage was preceded by a 5-month period of 324 millimeters $(\mathrm{mm})$ of precipitation (October 2004 through February 2005, at Site 1), well above the 1994-2006 5-month running average of 84 mm per month (Fransioli, 2007). Oliver and Whelan (2006) analyzed samples of this seepage for major ions, trace metals, and oxygen $(\mathrm{O})$ and hydrogen $(\mathrm{H})$ isotopic compositions in order to characterize the water and assess its interaction with the overlying soils and tuffs, or with anthropogenic materials used in the construction of the ESF. Corresponding baseline isotopic and chemical analyses of the precipitation inputs to the infiltration event(s) that produced the seepage were not available to Oliver and Whelan (2006) to estimate travel time, extent of water-rock interaction, or evaporative water loss during percolation through the unsaturated zone to aid further understanding of the hydrogeology at Yucca Mountain. 


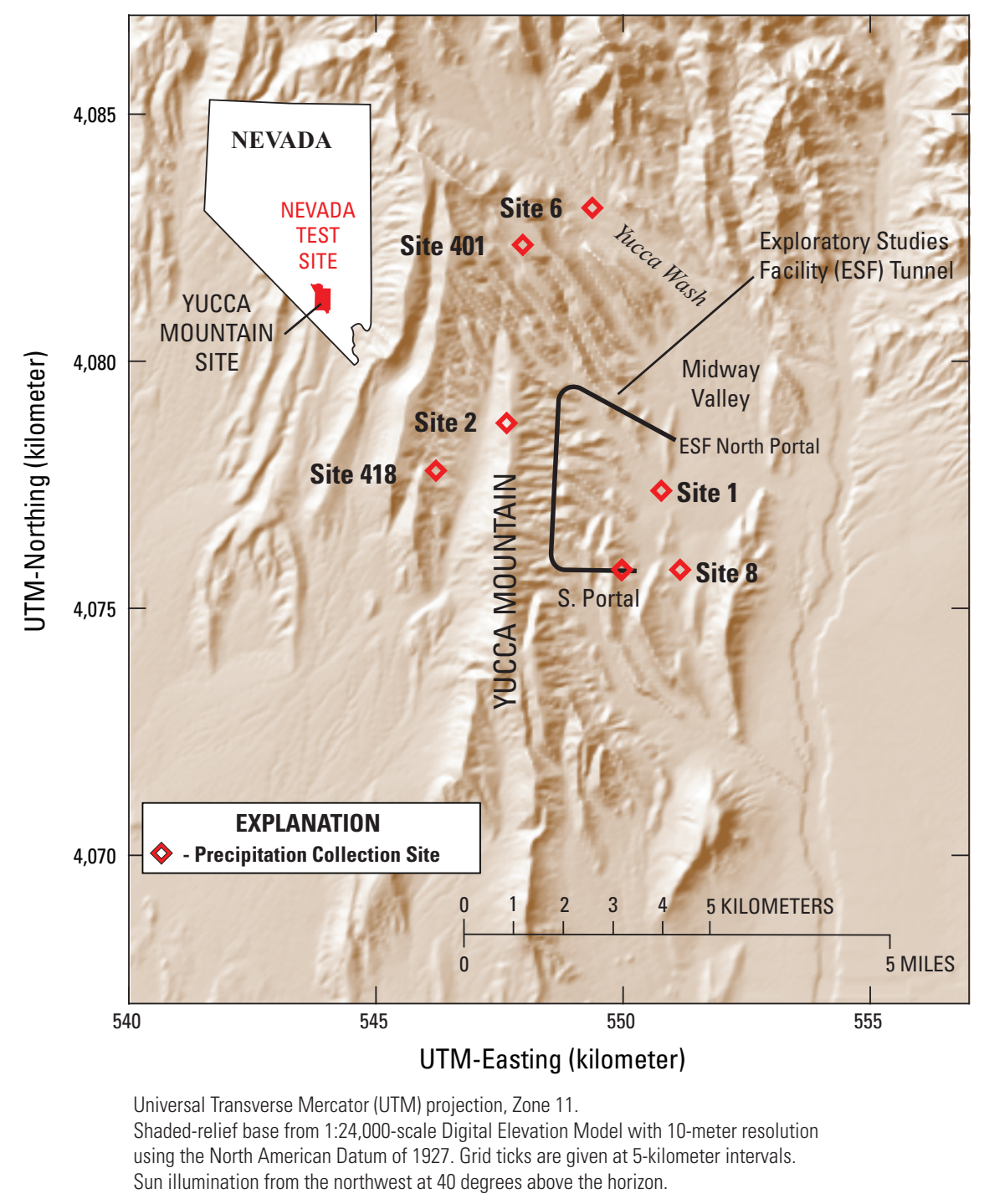

Figure 1. Shaded relief map of Yucca Mountain, Nevada, showing precipitation collection sites and the Exploratory Studies Facility.

One year later, in February 2006, a network of meteorological collection stations was installed (fig. 1) for chemical (major cations and anions, including short-chain organic acids and nutrients), delta oxygen-18 $\left(\delta^{18} \mathrm{O}\right)$, and delta deuterium $(\delta \mathrm{D})$ precipitation collection for analyses to facilitate interpretation of data from future seepage events. This report summarizes the chemical and isotopic compositions of modern precipitation samples collected from those stations between February 2006 and January 2009. These data complement previous chemical and isotopic data compiled for precipitation in the region (Milne and others, 1987; Benson and Klieforth, 1989; Ingraham and others, 1990; Friedman and others, 1992; Moscati and others, 2008).

\section{Equipment and Methods}

\section{Field Collection}

The precipitation collection network consists of seven surface sampling stations adjacent to Yucca Mountain (fig. 1). The stations range in elevation from $1,131 \mathrm{~m}$ at Site 8 (near the south portal of the ESF) to $1,562 \mathrm{~m}$ at Site 401 (near the north end of the crest of Yucca Mountain) (table 1). Each site has two collectors for precipitation samples: one for isotope analyses and one for chemistry analyses. Samples for isotope 
Table 1. Location of precipitation collection stations at Yucca Mountain, Nevada.

[Station coordinates are taken from the Universal Tranverse Mercator (UTM) projection, Zone 11, using the North American Datum of 1927 for elevations; deg $^{\circ}$ min' sec", degrees, minutes, seconds]

\begin{tabular}{lccccc}
\hline $\begin{array}{c}\text { Station } \\
\text { name }\end{array}$ & $\begin{array}{c}\text { Elevation } \\
\text { meters }\end{array}$ & $\begin{array}{c}\text { North Latitude } \\
\text { deg }^{\circ} \text { min' sec" }^{\circ}\end{array}$ & $\begin{array}{c}\text { West Longitude } \\
\text { deg }^{\circ} \text { min' sec" }^{\prime \prime}\end{array}$ & $\begin{array}{c}\text { UTM-Northing } \\
\text { meters }\end{array}$ & $\begin{array}{c}\text { UTM-Easting } \\
\text { meters }\end{array}$ \\
\hline Site 1 & 1,143 & $36^{\circ} 50^{\prime} 34^{\prime \prime}$ & $116^{\circ} 25^{\prime} 50^{\prime \prime}$ & 550784 & 4077374 \\
Site 2 & 1,478 & $36^{\circ} 51^{\prime} 19^{\prime \prime}$ & $116^{\circ} 27^{\prime} 56^{\prime \prime}$ & 547646 & 4078753 \\
Site 6 & 1,315 & $36^{\circ} 53^{\prime} 40^{\prime \prime}$ & $116^{\circ} 26^{\prime} 45^{\prime \prime}$ & 549388 & 4083097 \\
Site 8 & 1,131 & $36^{\circ} 49^{\prime} 42^{\prime \prime}$ & $116^{\circ} 25^{\prime} 35^{\prime \prime}$ & 551161 & 4075773 \\
Site 401 & 1,562 & $36^{\circ} 53^{\prime} 16^{\prime \prime}$ & $116^{\circ} 27^{\prime} 42^{\prime \prime}$ & 547995 & 4082387 \\
Site 418 & 1,301 & $36^{\circ} 50^{\prime} 48^{\prime \prime}$ & $116^{\circ} 28^{\prime} 54 "$ & 546213 & 4077785 \\
South Portal & 1,198 & $36^{\circ} 49^{\prime} 42^{\prime \prime}$ & $116^{\circ} 26^{\prime} 23^{\prime \prime}$ & 549965 & 4075773 \\
\hline
\end{tabular}

analyses are collected in a standard 4-in. [about 10-centimeter $(\mathrm{cm})$ ] diameter, plastic NovaLynx ${ }^{\mathrm{TM}}$ rain gauge (fig. 2A). The inner cylinder of the rain gauge, with a capacity of 1-in. (about $25 \mathrm{~mm}$ ) of rainfall, contains approximately 5 millimeter $(\mathrm{mL})$ of petroleum-based oil to retard evaporation of collected water. The top of the collector is fitted with a chemically inert mesh to prevent insects (which are attracted to the oil) from entering the collector, and with vertical plastic cable ties (see fig. 2A) to limit bird perching. During sampling, water is withdrawn from the collector using a syringe fitted with a smalldiameter, flexible tube.

The collector for samples for chemistry analyses, modeled after a design described by Milne and others (1987) and Claassen and Halm (1994), is a 30.5-cm-diameter, heavywalled polyvinylchloride (PVC) pipe (fig. 2B). The height of the cylinder is approximately $2 \mathrm{~m}$, of which about $30 \mathrm{~cm}$ are buried to enhance stability. Secured with a hose clamp around the outside of the PVC pipe, a set of two nested, 6-mil, flat, polyethylene sample-collection bags $(76 \mathrm{~cm}$ long) are hung inside the cylinder, with the innermost bag having a 1- to 2-cm-diameter restriction placed near the middle, so that the bag takes an hourglass shape. Double bagging ensures against samples being lost due to leakage, and the constriction of the bag minimizes exposure to atmospheric vapor and evaporation. The outer bag also prevents soil condensation from forming on the inner bag. The height of the cylinder limits contamination from surface dust stirred up by motor vehicles, wildlife, and wind. A precipitation sample was collected by removing the inner bag, snipping a lower corner with scissors, and draining the water into a sample bottle. The chemistry and isotope sample collectors both typically were sampled, emptied, and serviced within 24 to 48 hours after the cessation of a precipitation event. There was no filtering of the samples.

The initial network was installed in February 2006, with one collector at each station. After several months of sample chemistry analyses (cation and anion analyses by ion chromatography), it became apparent that the presence of insects and residual oil in the collector were contaminating the samples (giving excessive organic and sulfate content) and limiting the usefulness of the chemistry data (Moscati and others, 2008). Therefore, a second set of oil-free collectors was installed at each site, and collection from the new collectors commenced in December 2007. The initial collector at each site continued to be used as the isotope sample collector because field experiments and results described by Friedman and others (1992) showed that the use of oil to prevent evaporation did not affect the isotope analyses. The chemistry data presented in this paper were obtained from this second set of collectors. Sampling Site 418 was eliminated from the network after the September 25, 2007 collection because of a permanent gate closure on the Nevada Test Site.

\section{Major Cation and Anion Analysis}

Major cations and anions were analyzed, respectively, by two ion chromatographs. In either case, an aliquot of 500 microliter $(\mu \mathrm{L})$ of sample was introduced into the instrument. The sample was then separated and measured by use of a system consisting of a guard column, analytical column, suppressor device, and conductivity detector (O'Dell and others, 1984). The instrument software automatically calculated analyte concentrations from the sample and reported results in milligrams per liter $(\mathrm{mg} / \mathrm{L})$. Major-ion measurements of individual chemistry samples are \pm 15 percent or better (2-sigma error), except for bicarbonate $\left(\mathrm{HCO}_{3}\right)$, which is \pm 20 percent. That estimate of accuracy is based on the deviation of the measured values compared to accepted values for prepared standards for each analyte. Hydrogen activity $(\mathrm{pH})$ was analyzed in the laboratory with a compact $\mathrm{pH}$ meter requiring approximately $0.5 \mathrm{~mL}$ of sample for analysis. The resolution of $\mathrm{pH}$ measurements is 0.01 . 

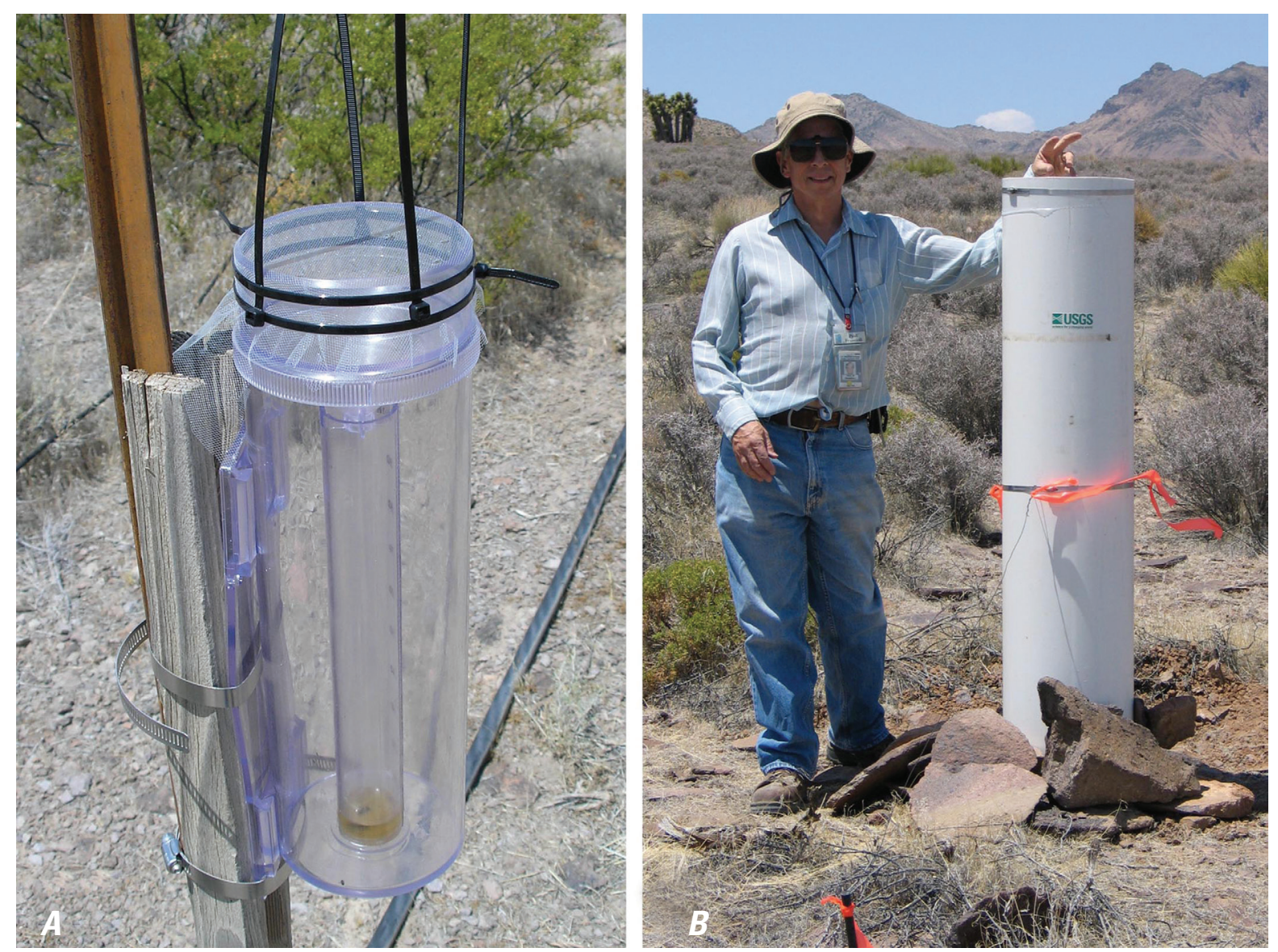

Figure 2. Precipitation collectors for isotope and chemistry samples at Yucca Mountain, Nevada.

\section{Stable Isotopic Analysis}

Deuterium (D) analyses were made after a $2-\mu \mathrm{L}$ aliquot of sample was converted to hydrogen gas by reaction with zinc metal shavings at $500^{\circ} \mathrm{C}$ (Kendall and Coplen, 1985). Magnetic-sector mass spectrometry was used to measure the deuterium content of the hydrogen gas, to separate the $\mathrm{HD}^{+}$from $\mathrm{H} 2$, to determine the ratio of the two ions, and to compare the ratio to that in a known sample of hydrogen gas. The results are reported in delta $(\delta)$ notation as the per mil (\%o) deviation relative to Vienna Standard Mean Ocean Water (VSMOW):

$$
\delta \mathrm{D}(\% 0)=\left[\left(\mathrm{R}_{\text {sample }}-\mathrm{R}_{\text {standard }}\right) /\left(\mathrm{R}_{\text {standard }}\right)\right] 1000,
$$

where $\mathrm{R}_{\text {sample }}$ and $\mathrm{R}_{\text {standard }}$ refer to the ratio of deuterium to hydrogen in the sample and standard (VSMOW), respectively. Samples were run in replicate. The $\delta \mathrm{D}$ values are precise to $\pm 2 \%$ (2-sigma error).

Oxygen-18 $\left({ }^{18} \mathrm{O}\right)$ analyses were made after a $200-\mu \mathrm{L}$ aliquot of sample was equilibrated with carbon dioxide $\left(\mathrm{CO}_{2}\right)$ of known oxygen isotopic composition at $40^{\circ} \mathrm{C}$ for at least
9 hours. The gas was then drawn through the Multiprep ${ }^{\mathrm{TM}}$ device where water was removed by passage through a cryogenic trap at dry-ice temperature $\left(-78^{\circ} \mathrm{C}\right)$. The dried $\mathrm{CO}_{2}$ was then analyzed for its $\delta^{18} \mathrm{O}$ by magnetic-sector mass spectrometry. The results are reported in $\delta$ notation in \%o units relative to VSMOW:

$$
\delta^{18} 0(\% 0)=\left[\left(R_{\text {sample }}-R_{\text {standard }}\right) /\left(R_{\text {standard }}\right)\right] 1000,
$$

where $\mathrm{R}_{\text {sample }}$ and $\mathrm{R}_{\text {standard }}$ refer to the ratio of oxygen-18 to oxygen-16 in the sample and standard, respectively. The $\delta^{18} \mathrm{O}$ values are precise to $\pm 0.2 \%$ o (2-sigma error).

\section{Major Cation and Anion Chemistry}

Major cation and anion concentrations, calculated total dissolved solids (TDS), and calculated conductivity of 75 precipitation samples collected from December 2007 to January 2009 are reported in figure 3 and in table 2 . The waters are relatively dilute, with resulting poor ionic charge balance. 


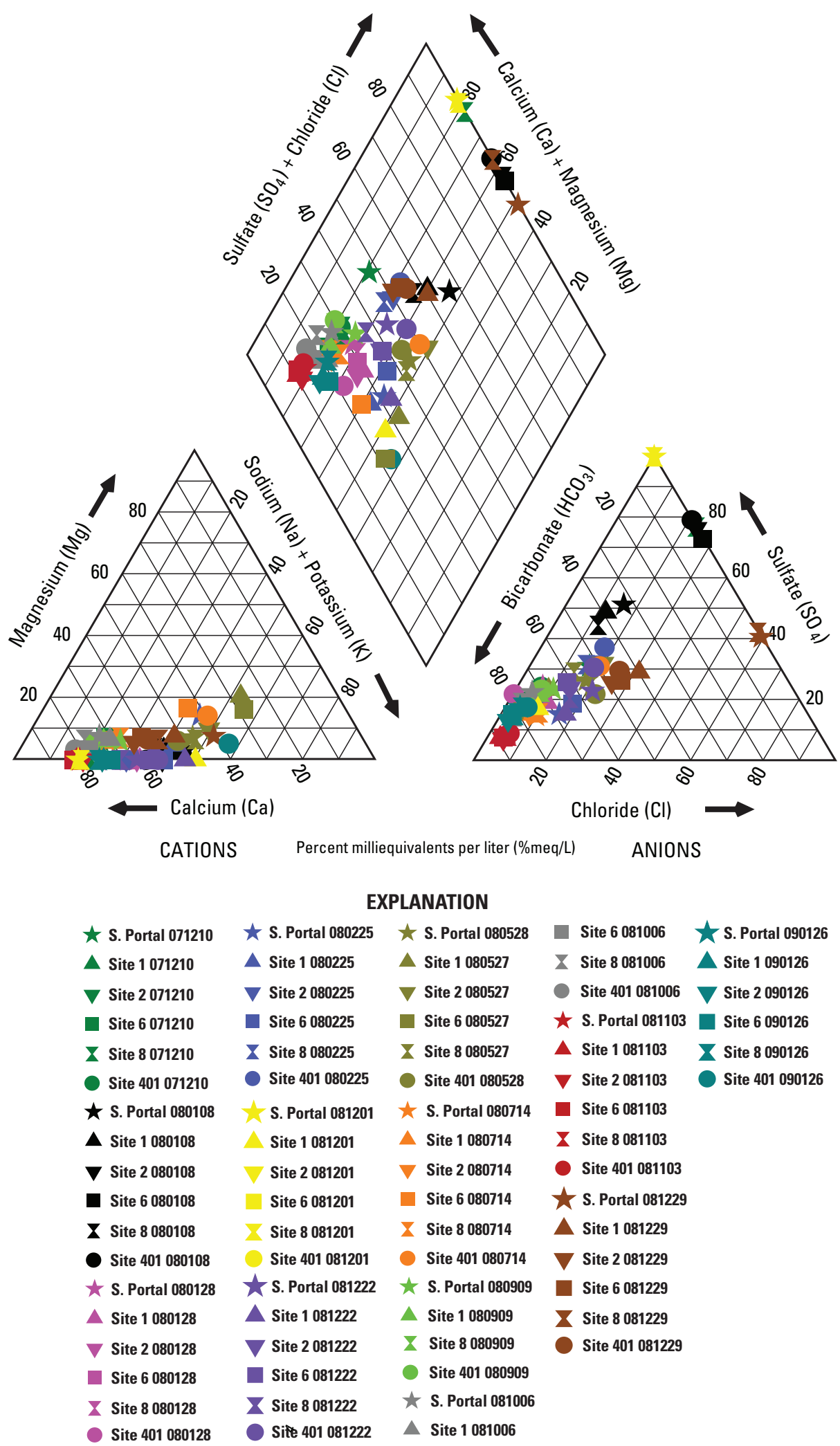

Sample name contains collection date in "yymmdd" (year/month/day) format.

Figure 3. Relative concentrations on piper diagram, in percent milliequivalents per liter, of the major ions in precipitation samples from monitoring stations at Yucca Mountain, Nevada, December 2007 to January 2009. 
Table 2. Major cation and anion concentrations for precipitation samples at Yucca Mountain, Nevada, December 2007 to January 2009.

[Abbreviations: mg/L, milligrams per liter; TDS, total dissolved solids; $\mu \mathrm{S} / \mathrm{cm}$, microsiemens per centimeter; NA, not available. TDS and conductivity are based on calculations from ion concentrations, not field measurements. Sample name contains collection date format "yy/ $\mathrm{mm} / \mathrm{dd} "$ (year/month/day)]

\begin{tabular}{|c|c|c|c|c|c|c|c|c|c|}
\hline Sample name & $\begin{array}{c}\mathrm{Na} \\
\mathrm{mg} / \mathrm{L}\end{array}$ & $\begin{array}{c}\mathrm{NH}_{4} \\
\mathrm{mg} / \mathrm{L}\end{array}$ & $\begin{array}{c}\mathrm{Mg} \\
\mathrm{mg} / \mathrm{L}\end{array}$ & $\begin{array}{c}\mathrm{K} \\
\mathrm{mg} / \mathrm{L}\end{array}$ & $\begin{array}{c}\mathrm{Ca} \\
\mathrm{mg} / \mathrm{L}\end{array}$ & $\begin{array}{c}F \\
\mathrm{mg} / \mathrm{L}\end{array}$ & $\begin{array}{c}\text { Acetate } \\
\mathrm{mg} / \mathrm{L}\end{array}$ & $\begin{array}{c}\text { Formate } \\
\mathrm{mg} / \mathrm{L}\end{array}$ & $\begin{array}{c}\text { Propionate } \\
\text { mg/L }\end{array}$ \\
\hline S. Portal 071210 & 0.27 & 0.53 & 0.05 & 0.06 & 0.93 & $<0.01$ & $<0.01$ & $<0.01$ & $<0.14$ \\
\hline Site 1071210 & 0.31 & 0.54 & 0.05 & 0.14 & 0.95 & $<0.01$ & $<0.01$ & $<0.01$ & $<0.14$ \\
\hline Site 2071210 & 0.46 & 0.87 & 0.08 & 0.09 & 1.57 & $<0.01$ & $<0.01$ & $<0.01$ & $<0.14$ \\
\hline Site 6071210 & 0.30 & 0.61 & 0.06 & 0.06 & 0.96 & $<0.01$ & $<0.01$ & $<0.01$ & $<0.14$ \\
\hline Site 8071210 & 0.21 & 0.49 & $<0.05$ & 0.04 & 0.73 & $<0.01$ & $<0.01$ & $<0.01$ & $<0.14$ \\
\hline Site 401071210 & 0.49 & 0.96 & 0.09 & 0.11 & 1.59 & $<0.01$ & $<0.01$ & $<0.01$ & $<0.14$ \\
\hline S. Portal 080108 & 1.40 & 0.49 & 0.06 & 0.27 & 1.47 & $<0.01$ & $<0.01$ & $<0.01$ & $<0.14$ \\
\hline Site 1080108 & 1.02 & 0.38 & 0.05 & 0.06 & 1.31 & $<0.01$ & $<0.01$ & $<0.01$ & $<0.14$ \\
\hline Site 2080108 & 0.74 & 0.28 & $<0.05$ & 0.04 & 0.94 & $<0.01$ & $<0.01$ & $<0.01$ & $<0.14$ \\
\hline Site 6080108 & 1.17 & 0.75 & 0.06 & 0.40 & 1.47 & $<0.01$ & $<0.01$ & $<0.01$ & $<0.14$ \\
\hline Site 8080108 & 1.28 & 0.48 & 0.07 & 0.07 & 1.87 & $<0.01$ & $<0.01$ & $<0.01$ & $<0.14$ \\
\hline Site 401080108 & 0.70 & 0.41 & $<0.05$ & $<0.04$ & 1.05 & $<0.01$ & $<0.01$ & $<0.01$ & $<0.14$ \\
\hline S. Portal 080128 & 0.17 & 0.28 & $<0.05$ & $<0.04$ & 0.39 & $<0.01$ & $<0.01$ & $<0.01$ & $<0.15$ \\
\hline Site 1080128 & 0.20 & 0.27 & $<0.05$ & 0.08 & 0.40 & $<0.01$ & $<0.01$ & $<0.01$ & $<0.15$ \\
\hline Site 2080128 & 0.16 & 0.28 & $<0.05$ & 0.06 & 0.33 & $<0.01$ & $<0.01$ & $<0.01$ & $<0.15$ \\
\hline Site 6080128 & 0.16 & 0.24 & $<0.05$ & $<0.04$ & 0.30 & $<0.01$ & $<0.01$ & $<0.01$ & $<0.15$ \\
\hline Site 8080128 & 0.16 & 0.25 & $<0.05$ & $<0.04$ & 0.32 & $<0.01$ & $<0.01$ & $<0.01$ & $<0.15$ \\
\hline Site 401080128 & 0.15 & 0.24 & $<0.05$ & $<0.04$ & 0.28 & $<0.01$ & $<0.01$ & $<0.01$ & $<0.15$ \\
\hline S. Portal 080225 & 2.55 & 4.18 & 0.51 & 0.81 & 2.42 & $<0.01$ & $<0.01$ & $<0.01$ & $<0.15$ \\
\hline Site 1080225 & 1.12 & 3.92 & 0.11 & 1.37 & 2.09 & $<0.01$ & $<0.01$ & $<0.01$ & $<0.15$ \\
\hline Site 2080225 & 0.91 & 1.62 & $<0.05$ & 0.22 & 1.99 & $<0.01$ & $<0.01$ & $<0.01$ & $<0.15$ \\
\hline Site 6080225 & 1.15 & 1.72 & $<0.05$ & 0.47 & 1.73 & $<0.01$ & $<0.01$ & $<0.01$ & $<0.15$ \\
\hline Site 8080225 & 0.90 & 1.04 & $<0.05$ & 0.12 & 2.01 & $<0.01$ & $<0.01$ & $<0.01$ & $<0.15$ \\
\hline Site 401080225 & 0.72 & 1.31 & $<0.05$ & 0.12 & 1.53 & $<0.01$ & $<0.01$ & $<0.01$ & $<0.15$ \\
\hline S. Portal 080528 & 6.16 & 4.70 & 0.59 & 1.61 & 6.27 & 0.02 & $<0.01$ & 0.02 & $<0.15$ \\
\hline Site 1080527 & 8.45 & 30.4 & 4.46 & 22.4 & 9.71 & 0.02 & 0.04 & 0.06 & 0.16 \\
\hline Site 2080527 & 6.53 & 0.49 & 1.11 & 6.25 & 7.36 & 0.03 & 8.90 & $<0.01$ & 1.77 \\
\hline Site 6080527 & 6.76 & 34.3 & 3.51 & 26.3 & 9.87 & 0.02 & 0.02 & 0.04 & 0.68 \\
\hline Site 8080527 & 8.72 & 4.04 & 0.77 & 2.69 & 8.82 & 0.02 & $<0.01$ & 0.02 & $<0.15$ \\
\hline Site 401080528 & 3.57 & 2.49 & 0.34 & 1.40 & 4.62 & 0.01 & $<0.01$ & 0.01 & $<0.15$ \\
\hline S. Portal 080714 & 0.84 & 1.09 & 0.14 & 0.44 & 3.33 & $<0.01$ & 0.12 & 0.11 & $<0.15$ \\
\hline Site 1080714 & 0.68 & 1.46 & 0.14 & 0.73 & 2.70 & $<0.01$ & 0.17 & 0.14 & $<0.15$ \\
\hline Site 2080714 & 0.98 & 2.59 & 0.24 & 0.95 & 3.85 & $<0.01$ & 0.04 & 0.03 & $<0.15$ \\
\hline Site 6080714 & 2.48 & 13.9 & 2.09 & 11.8 & 8.84 & 0.01 & 0.04 & 0.10 & 0.30 \\
\hline Site 8080714 & 1.41 & 1.79 & 0.31 & 1.10 & 4.61 & $<0.01$ & $<0.01$ & 0.03 & $<0.15$ \\
\hline Site 401080714 & 2.99 & 12.1 & 1.70 & 12.6 & 7.57 & 0.01 & 0.67 & 0.20 & $<0.15$ \\
\hline
\end{tabular}


Table 2. Major cation and anion concentrations for precipitation samples at Yucca Mountain, Nevada, December 2007 to January 2009.-Continued

[Abbreviations: $\mathrm{mg} / \mathrm{L}$, milligrams per liter; TDS, total dissolved solids; $\mu \mathrm{S} / \mathrm{cm}$, microsiemens per centimeter; NA, not available. TDS and conductivity are based on calculations from ion concentrations, not field measurements. Sample name contains collection date format "yy/mm/dd" (year/month/day)]

\begin{tabular}{|c|c|c|c|c|c|c|c|c|c|c|}
\hline Sample name & $\underset{\mathrm{mg} / \mathrm{L}}{\mathrm{Cl}}$ & $\begin{array}{l}\mathrm{NO}_{2} \\
\mathrm{mg} / \mathrm{L}\end{array}$ & $\begin{array}{l}\mathrm{HCO}_{3} \\
\mathrm{mg} / \mathrm{L}\end{array}$ & $\begin{array}{c}\mathrm{SO}_{4} \\
\mathrm{mg} / \mathrm{L}\end{array}$ & $\begin{array}{c}\mathrm{Br} \\
\mathrm{mg} / \mathrm{L}\end{array}$ & $\begin{array}{l}\mathrm{NO}_{3} \\
\mathrm{mg} / \mathrm{L}\end{array}$ & $\begin{array}{l}\mathrm{PO}_{4} \\
\mathrm{mg} / \mathrm{L}\end{array}$ & $\begin{array}{l}\text { TDS } \\
\mathrm{mg} / \mathrm{L}\end{array}$ & $\begin{array}{c}\text { Conductivity } \\
\mu \mathrm{S} / \mathrm{cm}\end{array}$ & Water-type \\
\hline S. Portal 071210 & 0.22 & $<0.13$ & 1.20 & 0.56 & $<0.03$ & 2.02 & $<0.02$ & 5.8 & 10 & $\mathrm{Ca}-\mathrm{NO}_{3}$ \\
\hline Site 1071210 & 0.14 & $<0.13$ & 2.40 & 0.60 & 0.08 & 2.00 & 0.10 & 7.3 & 11 & $\mathrm{Ca}-\mathrm{HCO}_{3}$ \\
\hline Site 2071210 & 0.20 & $<0.13$ & 3.40 & 0.94 & $<0.03$ & 3.27 & $<0.02$ & 11 & 17 & $\mathrm{Ca}-\mathrm{HCO}_{3}$ \\
\hline Site 6071210 & 0.13 & $<0.13$ & 3.80 & 0.79 & $<0.03$ & 3.30 & $<0.02$ & 10 & 14 & $\mathrm{Ca}-\mathrm{HCO}_{3}$ \\
\hline Site 8071210 & 0.11 & $<0.13$ & $<1.2$ & 0.50 & $<0.03$ & 1.79 & $<0.02$ & 3.9 & 7.7 & $\mathrm{Ca}-\mathrm{NO}_{3}$ \\
\hline Site 401071210 & 0.20 & $<0.13$ & 3.70 & 1.03 & $<0.03$ & 4.29 & $<0.02$ & 12 & 19 & $\mathrm{Ca}-\mathrm{NO}_{3}$ \\
\hline S. Portal 080108 & 0.42 & $<0.13$ & 1.50 & 1.84 & $<0.03$ & 1.80 & $<0.02$ & 9.3 & 17 & $\mathrm{Ca}-\mathrm{SO}_{4}$ \\
\hline Site 1080108 & 0.27 & $<0.13$ & 1.50 & 1.49 & $<0.03$ & 1.74 & $<0.02$ & 7.8 & 14 & $\mathrm{Ca}-\mathrm{SO}_{4}$ \\
\hline Site 2080108 & 0.22 & $<0.13$ & $<1.2$ & 0.97 & $<0.03$ & 1.38 & $<0.02$ & 4.6 & 9.1 & $\mathrm{Ca}-\mathrm{NO}_{3}$ \\
\hline Site 6080108 & 0.34 & $<0.13$ & $<1.2$ & 1.25 & 0.14 & 2.18 & $<0.02$ & 7.8 & 16 & $\mathrm{Ca}-\mathrm{NO}_{3}$ \\
\hline Site 8080108 & 0.36 & $<0.13$ & 2.24 & 1.84 & $<0.03$ & 2.22 & $<0.02$ & 10 & 18 & $\mathrm{Ca}-\mathrm{SO}_{4}$ \\
\hline Site 401080108 & 0.21 & $<0.13$ & $<1.2$ & 1.10 & $<0.03$ & 2.65 & $<0.02$ & 6.1 & 11 & $\mathrm{Ca}-\mathrm{NO}_{3}$ \\
\hline S. Portal 080128 & 0.10 & $<0.13$ & 1.7 & 0.48 & $<2.0$ & 1.03 & $<2.0$ & 4.2 & 6.0 & $\mathrm{Ca}-\mathrm{HCO}_{3}$ \\
\hline Site 1080128 & 0.14 & $<0.13$ & 1.6 & 0.35 & $<2.0$ & 1.02 & $<2.0$ & 4.0 & 6.0 & $\mathrm{Ca}-\mathrm{HCO}_{3}$ \\
\hline Site 2080128 & 0.12 & $<0.13$ & 1.7 & 0.35 & $<2.0$ & 1.01 & $<2.0$ & 4.0 & 5.7 & $\mathrm{Ca}-\mathrm{HCO}_{3}$ \\
\hline Site 6080128 & 0.10 & $<0.13$ & 1.4 & 0.33 & $<2.0$ & 0.99 & $<2.0$ & 3.5 & 5.1 & $\mathrm{Ca}-\mathrm{HCO}_{3}$ \\
\hline Site 8080128 & 0.10 & $<0.13$ & 1.3 & 0.33 & $<2.0$ & 0.88 & $<2.0$ & 3.3 & 5.0 & $\mathrm{Ca}-\mathrm{HCO}_{3}$ \\
\hline Site 401080128 & $<0.09$ & $<0.13$ & 1.7 & 0.38 & $<2.0$ & 0.92 & $<2.0$ & 3.7 & 5.0 & $\mathrm{Ca}-\mathrm{HCO}_{3}$ \\
\hline S. Portal 080225 & 2.61 & $<0.13$ & 19 & 3.37 & $<2.0$ & 4.06 & $<2.0$ & 40 & 60 & $\mathrm{NH}_{4}-\mathrm{HCO}_{3}$ \\
\hline Site 1080225 & 0.60 & $<0.13$ & 13 & 2.98 & $<2.0$ & 3.46 & 3.85 & 33 & 45 & $\mathrm{NH}_{4}-\mathrm{HCO}_{3}$ \\
\hline Site 2080225 & 0.69 & $<0.13$ & 3.4 & 1.74 & $<2.0$ & 4.92 & $<2.0$ & 15 & 26 & $\mathrm{Ca}-\mathrm{NO}_{3}$ \\
\hline Site 6080225 & 1.14 & $<0.13$ & 6.9 & 1.62 & $<2.0$ & 3.88 & $<2.0$ & 19 & 29 & $\mathrm{NH}_{4}-\mathrm{HCO}_{3}$ \\
\hline Site 8080225 & 0.61 & $<0.13$ & 3.6 & 1.69 & $<2.0$ & 3.39 & $<2.0$ & 13 & 22 & $\mathrm{Ca}-\mathrm{HCO}_{3}$ \\
\hline Site 401080225 & 0.49 & $<0.13$ & 2.2 & 1.43 & $<2.0$ & 4.01 & $<2.0$ & 12 & 21 & $\mathrm{Ca}-\mathrm{NO}_{3}$ \\
\hline S. Portal 080528 & 4.27 & $<0.13$ & 23 & 8.65 & $<2.0$ & 8.35 & $<2.0$ & 64 & 99 & $\mathrm{Ca}-\mathrm{HCO}_{3}$ \\
\hline Site 1080527 & 9.43 & $<0.13$ & 121 & 33.7 & $<2.0$ & 0.08 & 36.6 & 276 & 338 & $\mathrm{NH}_{4}-\mathrm{HCO}_{3}$ \\
\hline Site 2080527 & 4.76 & $<0.13$ & 20 & 10.5 & $<2.0$ & 0.11 & $<2.0$ & 57 & 90 & $\mathrm{Ca}-\mathrm{HCO}_{3}$ \\
\hline Site 6080527 & 6.20 & 1.87 & 149 & 24.5 & $<2.0$ & 0.96 & 20.2 & 285 & 355 & $\mathrm{NH}_{4}-\mathrm{HCO}_{3}$ \\
\hline Site 8080527 & 4.09 & 0.45 & 31 & 12.4 & $<2.0$ & 11.0 & $<2.0$ & 84 & 124 & $\mathrm{Ca}-\mathrm{HCO}_{3}$ \\
\hline Site 401080528 & 3.31 & $<0.13$ & 14 & 4.36 & $<2.0$ & 5.85 & $<2.0$ & 40 & 63 & $\mathrm{Ca}-\mathrm{HCO}_{3}$ \\
\hline S. Portal 080714 & 0.72 & 0.13 & 9.3 & 1.45 & $<2.0$ & 2.46 & $<2.0$ & 20 & 30 & $\mathrm{Ca}-\mathrm{HCO}_{3}$ \\
\hline Site 1080714 & 0.58 & $<0.13$ & 8.5 & 1.35 & $<2.0$ & 2.82 & $<2.0$ & 19 & 29 & $\mathrm{Ca}-\mathrm{HCO}_{3}$ \\
\hline Site 2080714 & 0.81 & $<0.13$ & 14 & 2.22 & $<2.0$ & 3.26 & $<2.0$ & 29 & 44 & $\mathrm{Ca}-\mathrm{HCO}_{3}$ \\
\hline Site 6080714 & 2.97 & 4.56 & 56 & 10.3 & $<2.0$ & 3.13 & 17.2 & 133 & 167 & $\mathrm{NH}_{4}-\mathrm{HCO}_{3}$ \\
\hline Site 8080714 & 1.14 & $<0.13$ & 14 & 2.41 & $<2.0$ & 4.16 & $<2.0$ & 31 & 46 & $\mathrm{Ca}-\mathrm{HCO}_{3}$ \\
\hline Site 401080714 & 5.19 & 5.38 & 23 & 11.4 & $<2.0$ & 0.08 & 22.8 & 105 & 140 & $\mathrm{NH}_{4}-\mathrm{PO}_{4}$ \\
\hline
\end{tabular}


Table 2. Major cation and anion concentrations for precipitation samples at Yucca Mountain, Nevada, December 2007 to January 2009.-Continued

[Abbreviations: $\mathrm{mg} / \mathrm{L}$, milligrams per liter; TDS, total dissolved solids; $\mu \mathrm{S} / \mathrm{cm}$, microsiemens per centimeter; NA, not available. TDS and conductivity are based on calculations from ion concentrations, not field measurements. Sample name contains collection date format "yy/ $\mathrm{mm} / \mathrm{dd}^{\prime}$ (year/month/day)]

\begin{tabular}{|c|c|c|c|c|c|c|c|c|c|}
\hline Sample name & $\begin{array}{c}\mathrm{Na} \\
\mathrm{mg} / \mathrm{L}\end{array}$ & $\begin{array}{l}\mathrm{NH}_{4} \\
\mathrm{mg} / \mathrm{L}\end{array}$ & $\begin{array}{c}\mathrm{Mg} \\
\mathrm{mg} / \mathrm{L}\end{array}$ & $\begin{array}{c}\mathrm{K} \\
\mathrm{mg} / \mathrm{L}\end{array}$ & $\begin{array}{c}\mathrm{Ca} \\
\mathrm{mg} / \mathrm{L}\end{array}$ & $\begin{array}{c}F \\
\mathrm{mg} / \mathrm{L}\end{array}$ & $\begin{array}{c}\text { Acetate } \\
\mathrm{mg} / \mathrm{L}\end{array}$ & $\begin{array}{c}\text { Formate } \\
\mathrm{mg} / \mathrm{L}\end{array}$ & $\begin{array}{c}\text { Propionate } \\
\mathrm{mg} / \mathrm{L}\end{array}$ \\
\hline S. Portal 080909 & 3.44 & 2.82 & 0.57 & 1.95 & 10.1 & 0.02 & 0.06 & 0.04 & $<0.15$ \\
\hline Site 1080909 & 2.25 & 1.79 & 0.46 & 1.49 & 8.91 & 0.02 & $<0.01$ & 0.01 & $<0.15$ \\
\hline Site 8080909 & 1.12 & 1.46 & 0.19 & 0.63 & 5.02 & $<0.01$ & 0.02 & 0.02 & $<0.15$ \\
\hline Site 401080909 & 1.56 & 2.16 & 0.33 & 0.79 & 6.99 & 0.01 & 0.02 & 0.01 & $<0.15$ \\
\hline S. Portal 081006 & 1.77 & 2.01 & 0.61 & 1.89 & 9.25 & 0.01 & 0.08 & 0.01 & $<0.15$ \\
\hline Site 1081006 & 0.99 & 2.45 & 0.30 & 1.66 & 5.10 & $<0.01$ & 0.03 & $<0.01$ & $<0.15$ \\
\hline Site 6081006 & 0.41 & 0.71 & 0.07 & 0.60 & 2.72 & $<0.01$ & 0.03 & $<0.01$ & $<0.15$ \\
\hline Site 8081006 & 0.92 & 1.04 & 0.31 & 0.80 & 5.77 & $<0.01$ & 0.07 & 0.02 & $<0.15$ \\
\hline Site 401081006 & 0.46 & 0.70 & 0.08 & 0.38 & 3.11 & $<0.01$ & 0.06 & 0.01 & $<0.15$ \\
\hline S. Portal 081103 & 0.14 & 0.25 & $<0.05$ & 0.11 & 0.80 & $<0.01$ & 0.07 & 0.03 & $<0.15$ \\
\hline Site 1081103 & 0.12 & 0.14 & $<0.05$ & 0.08 & 0.68 & $<0.01$ & 0.05 & 0.02 & $<0.15$ \\
\hline Site 2081103 & 0.20 & 0.46 & $<0.05$ & 0.18 & 1.11 & $<0.01$ & 0.05 & 0.01 & $<0.15$ \\
\hline Site 6081103 & 0.16 & 0.17 & $<0.05$ & 0.11 & 0.99 & $<0.01$ & 0.06 & 0.04 & $<0.15$ \\
\hline Site 8081103 & 0.13 & 0.15 & $<0.05$ & 0.07 & 0.67 & $<0.01$ & 0.03 & $<0.01$ & $<0.15$ \\
\hline Site 401081103 & 0.16 & 0.19 & $<0.05$ & 0.07 & 0.84 & $<0.01$ & 0.06 & 0.04 & $<0.15$ \\
\hline S. Portal 081201 & 0.06 & 0.13 & $<0.05$ & $<0.04$ & 0.24 & $<0.01$ & $<0.01$ & 0.03 & $<0.15$ \\
\hline Site 1081201 & 0.24 & 0.31 & $<0.05$ & 0.17 & 0.29 & $<0.01$ & 0.04 & $<0.01$ & $<0.15$ \\
\hline Site 2081201 & $<0.05$ & 0.12 & $<0.05$ & $<0.04$ & $<0.15$ & $<0.01$ & $<0.01$ & 0.04 & $<0.15$ \\
\hline Site 6081201 & $<0.05$ & 0.10 & $<0.05$ & $<0.04$ & $<0.15$ & $<0.01$ & $<0.01$ & 0.02 & $<0.15$ \\
\hline Site 8081201 & 0.05 & 0.11 & $<0.05$ & $<0.04$ & 0.19 & $<0.01$ & 0.02 & 0.01 & $<0.15$ \\
\hline Site 401081201 & $<0.05$ & 0.10 & $<0.05$ & $<0.04$ & $<0.15$ & $<0.01$ & $<0.01$ & 0.11 & $<0.15$ \\
\hline S. Portal 081222 & 0.38 & 0.44 & $<0.05$ & 0.05 & 0.69 & $<0.01$ & NA & $<0.01$ & $<0.15$ \\
\hline Site 1081222 & 0.49 & 0.37 & $<0.05$ & 0.24 & 0.61 & $<0.01$ & NA & $<0.01$ & $<0.15$ \\
\hline Site 2081222 & 0.87 & 0.46 & 0.05 & 0.15 & 1.35 & $<0.01$ & NA & $<0.01$ & $<0.15$ \\
\hline Site 6081222 & 0.60 & 0.25 & $<0.05$ & 0.06 & 0.93 & $<0.01$ & NA & $<0.01$ & $<0.15$ \\
\hline Site 8081222 & 0.34 & 0.40 & $<0.05$ & $<0.04$ & 0.70 & $<0.01$ & NA & $<0.01$ & $<0.15$ \\
\hline Site 401081222 & 0.59 & 0.29 & $<0.05$ & 0.07 & 0.82 & $<0.01$ & NA & $<0.01$ & $<0.15$ \\
\hline S. Portal 081229 & 0.73 & 0.61 & 0.06 & 0.05 & 0.52 & $<0.01$ & NA & $<0.01$ & $<0.15$ \\
\hline Site 1081229 & 0.69 & 0.55 & 0.07 & 0.05 & 0.80 & $<0.01$ & NA & $<0.01$ & $<0.15$ \\
\hline Site 2081229 & 0.74 & 0.66 & 0.08 & 0.07 & 1.42 & $<0.01$ & NA & $<0.01$ & $<0.15$ \\
\hline Site 6081229 & 0.80 & 0.73 & 0.10 & 0.07 & 1.41 & $<0.01$ & NA & $<0.01$ & $<0.15$ \\
\hline Site 8081229 & 0.70 & 0.50 & 0.07 & 0.05 & 0.96 & $<0.01$ & NA & 0.01 & $<0.15$ \\
\hline Site 401081229 & 0.92 & 0.78 & 0.10 & 0.07 & 1.47 & $<0.01$ & NA & $<0.01$ & $<0.15$ \\
\hline S. Portal 090126 & 0.23 & 0.70 & $<0.05$ & 0.05 & 0.73 & $<0.01$ & $<0.01$ & $<0.01$ & $<0.15$ \\
\hline Site 1090126 & 0.26 & 0.64 & $<0.05$ & 0.05 & 0.75 & $<0.01$ & 0.02 & 0.01 & $<0.15$ \\
\hline Site 2090126 & 0.20 & 0.57 & $<0.05$ & 0.05 & 0.61 & $<0.01$ & $<0.01$ & $<0.01$ & $<0.15$ \\
\hline Site 6090126 & 0.20 & 0.57 & $<0.05$ & 0.04 & 0.53 & $<0.01$ & $<0.01$ & $<0.01$ & $<0.15$ \\
\hline Site 8090126 & 0.23 & 0.66 & $<0.05$ & 0.04 & 0.72 & $<0.01$ & $<0.01$ & 0.11 & $<0.15$ \\
\hline Site 401090126 & 0.21 & 1.10 & 0.05 & 1.42 & 0.60 & $<0.01$ & $<0.01$ & $<0.01$ & $<0.15$ \\
\hline
\end{tabular}


Table 2. Major cation and anion concentrations for precipitation samples at Yucca Mountain, Nevada, December 2007 to January 2009.-Continued

[Abbreviations: $\mathrm{mg} / \mathrm{L}$, milligrams per liter; TDS, total dissolved solids; $\mu \mathrm{S} / \mathrm{cm}$, microsiemens per centimeter; NA, not available. TDS and conductivity are based on calculations from ion concentrations, not field measurements. Sample name contains collection date format "yy/mm/dd" (year/month/day)]

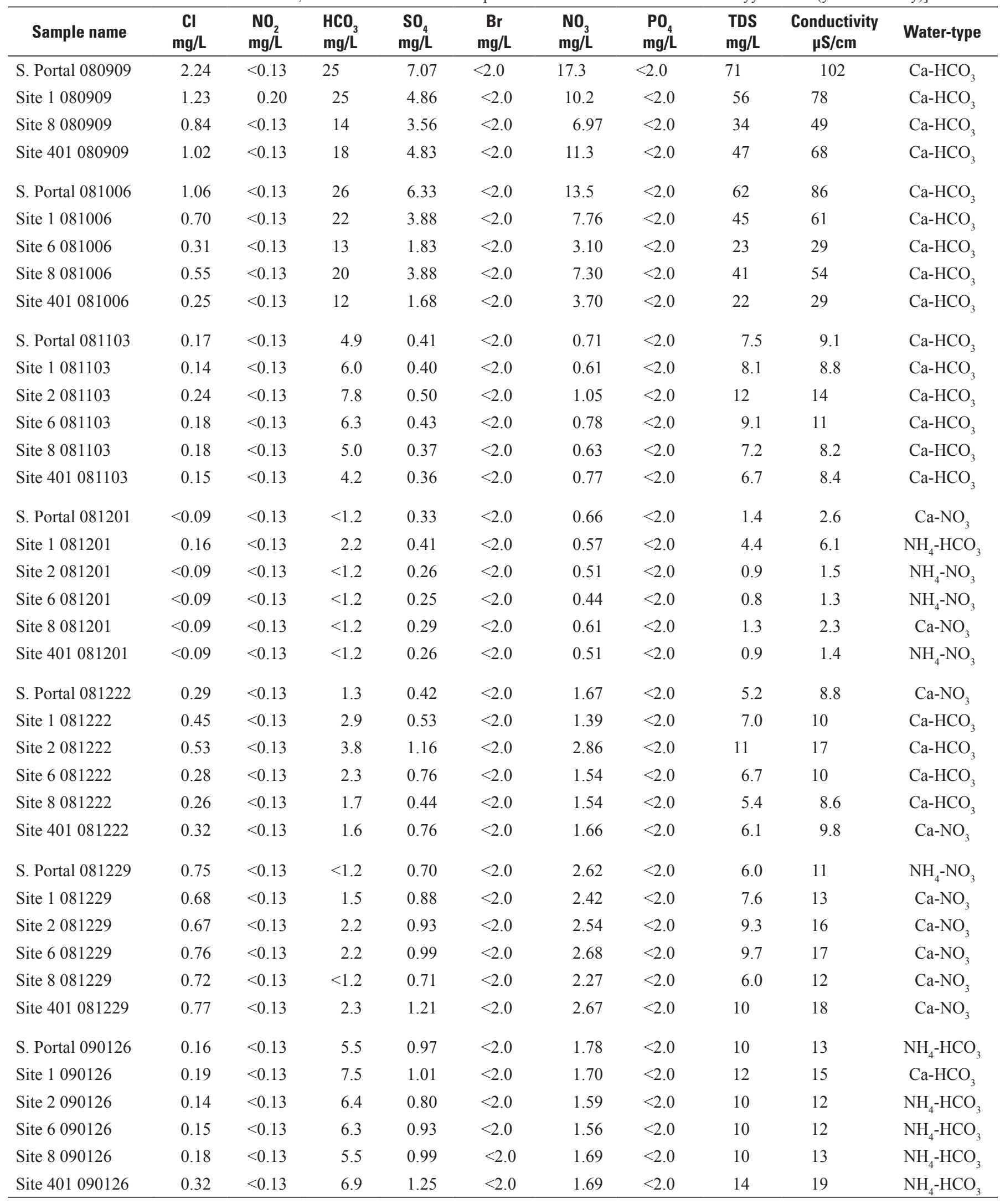


Dilution of these samples likely is a function of the length of their collection period, typically spanning several days of rainfall. Generally, rain samples collected at the beginning of an individual storm contain most of the soluble material available in the atmosphere, and consequently, rain toward the end of a storm is more dilute (Friedman and others, 2002). This effect is compounded because samples often accumulated over a period of many days, with associated intermittent storms (causing increased dilution), before collection.

The collected rainfall is slightly acidic, commonly with a $\mathrm{pH}$ between 6 and 7. The relatively high values of $\mathrm{pH}$ (compared to pure water in equilibrium with the atmosphere, $\mathrm{pH}$ 5.6 [Drever, 1997]) likely are not due to a lack of acidity in the precipitation but to the buffering by local, naturally alkaline dusts that contain large calcium-carbonate fractions.

The precipitation samples, defined on the basis of dissolved ions (after Back, 1966), commonly are characterized by calcium-bicarbonate-type water (fig. 3). A small percentage of other water types also are recognized, including calciumnitrate and ammonium-bicarbonate (23 percent and 16 percent, respectively, of the total samples analyzed). Eleven of the analyzed samples have low $\mathrm{HCO}_{3}$ values $(<1.2 \mathrm{mg} / \mathrm{L})$ and plot anomalously near the top-right part of the diamond on figure 3, accounting for almost half of the calcium-nitrate type waters identified.

Typical winter storm trajectories originating over the Pacific Ocean (Friedman and others, 1992) and tracking toward southern Nevada cross mountain ranges, playas, and alluvial fans. As these air masses move inland, terrestrial dust is entrained. That eolian dust, dominantly derived from arid soils, contains calcite $\left(\mathrm{CaCO}_{3}\right)$, salts (such as $\mathrm{NaCl}$ ), and gypsum $\left(\mathrm{CaSO}_{4} \cdot 2 \mathrm{H}_{2} \mathrm{O}\right)$ from pedogenic, authigenic, and playa settings (Reheis and Kihl, 1995) and is likely the main contributor of dissolved $\mathrm{Na}^{+}, \mathrm{Ca}^{2+}, \mathrm{Cl}^{-}$, and $\mathrm{SO}_{4}^{2-}$ to the meteoricprecipitation chemistry at Yucca Mountain. Elevated nitrate and ammonium concentrations may be both anthropogenic and natural (released by vegetation), but are more likely to be anthropogenic, linked to agricultural ammonium-nitrate fertilizers and animal urine (and to a lesser extent, vehicle emissions) in the nearby Amargosa River valley south and west of Yucca Mountain.

Oliver and Whelan (2006) reported the chemical characterization of the February 2005 seepage waters that triggered this study. They determined that those seepage waters, which traveled through the soil zone and through 40 to $80 \mathrm{~m}$ of Tiva Canyon Tuff, were of the calcium-sulfate-chloride type, which would plot in the top-center portion of the diamond in a Piper-type diagram (for example, see figure 3). Data presented here illustrate a marked shift in infiltration-water chemistry, from predominately calcium-bicarbonate type at the surface to calcium-sulfate-chloride type in the ESF, over a relatively short distance and percolation time. The Oliver and Whelan (2006) samples were collected from the floor of the ESF, on vents and rock bolts in the tunnel, and from exposed rock surfaces and fractures.

\section{Stable Isotopes}

The arid climate of Yucca Mountain has a mean annual precipitation of approximately $200 \mathrm{~mm}$ that occurs as convective precipitation in the summer monsoon period of July and August, or as lower-intensity, longer-duration periods of precipitation during the winter (Sharpe, 2007). Flint and others (2002) estimated that average net infiltration at Yucca Mountain is 3 percent of annual precipitation, or approximately 6 $\mathrm{mm} / \mathrm{year}$, and increases to approximately $20 \mathrm{~mm} /$ year at the exposed bedrock crest of Yucca Mountain. Precipitation and infiltration data at Yucca Mountain were used to estimate travel time, extent of water-rock interaction, and evaporative water loss during percolation through the unsaturated zone (Flint and others, 2002). Not only are estimates of infiltration important, but the stable-isotope chemistry of the precipitation also is needed to obtain baseline data for meteoric water prior to interaction with the soil or bedrock.

Thirty-six distinct precipitation events that occurred in 2006-09, with an average 3- to 4-day duration and an average $9.9 \mathrm{~mm}$ of accumulation, have been analyzed. Stable isotope data (more than 200 samples) from these events are summarized in table 3 and are represented on figure 4 as individual rainfall events designated by their date(s) of occurrence. Delta ${ }^{18} \mathrm{O}$ values range from 3.0 to $-20.4 \%$ and $\delta \mathrm{D}$ values range from 10 to $-147 \%$ and define a line of slope 6.4 on a $\delta \mathrm{D}$ $\delta^{18} \mathrm{O}$ plot, less than the slope of 8 of the Global Meteoric Water Line (GMWL). The GMWL, quantified by Craig (1961), is a relation between $\delta \mathrm{D}$ and $\delta^{18} \mathrm{O}$ values of meteoric waters, expressed as $\delta \mathrm{D}=8\left(\delta^{18} \mathrm{O}\right)+10$. The defined slope of Yucca Mountain precipitation (6.4) is the result of evaporation of falling raindrops (Benson and Klieforth, 1989), typical of arid environments. The ESF seepage samples collected in February 2005 exhibit a slope of 4 (fig. 4) and demonstrate continued evaporation as the water percolated through the bedrock or after it seeped into the tunnel (Oliver and Whelan, 2006).

There is a distinct seasonal difference in oxygen and deuterium isotope values (fig. 5). Although precipitation was collected and analyzed continually over the course of a year, precipitation that fell from November through April was labeled as winter precipitation, and precipitation that fell from May through October as summer precipitation. The average winter values are -11.9 and $-81 \%$, and for summer are -5.9 and $-51 \%$, resulting in an average difference of 6.0 and $30 \%$ for oxygen and deuterium, respectively. Weighted average values, where more influence is given to precipitation events of greater volume, for winter precipitation are -12.9 and $-93 \%$ and for summer precipitation are -9.9 and $-69 \%$ for oxygen and deuterium, respectively. The weighted averages equate to a seasonal difference of $3.0 \%$ for oxygen and $24 \%$ for deuterium. It should be noted that during the collection period of this study, precipitation that fell as snow was not differentiated from precipitation that fell as rain. Snow precipitation was rare, however, even in winter, and limited to high-altitude sites (for example, Sites 2 and 401). 
Table 3. Delta oxygen-18 $\left(\delta^{18} 0\right)$ and delta deuterium $(\delta D)$ values, and amounts, of precipitation samples at Yucca Mountain, Nevada, February 2006 to January 2009.

[Sample name contains collection date format "yy/mm/dd" (year/month/day); \%o, per mil; cm, centimeter; Precip. Amt., precipitation amount]

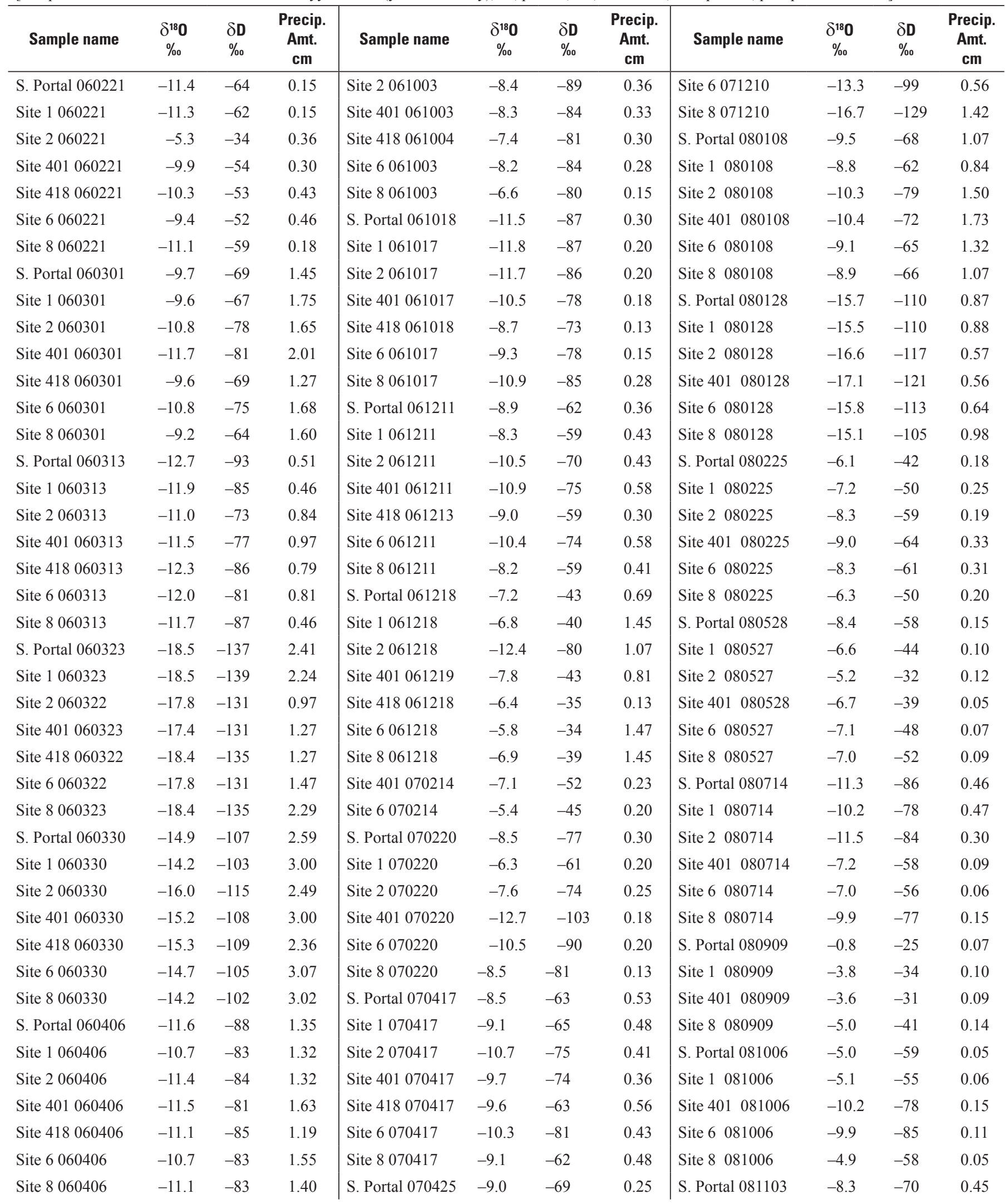


Table 3. Delta oxygen-18 $\left(\delta^{18} 0\right)$ and delta deuterium $(\delta D)$ values, and amounts, of precipitation samples at Yucca Mountain, Nevada, February 2006 to January 2009.-Continued

[Sample name contains collection date format "yy/mm/dd" (year/month/day); \%o, per mil; cm, centimeter; Precip. Amt., precipitation amount]

\begin{tabular}{|c|c|c|c|c|c|c|c|c|c|c|c|}
\hline Sample name & $\begin{array}{c}\delta^{180} \\
\% 0\end{array}$ & $\begin{array}{l}\delta \text { D } \\
\% 0\end{array}$ & $\begin{array}{c}\text { Precip. } \\
\text { Amt. } \\
\text { cm }\end{array}$ & Sample name & $\begin{array}{c}\delta^{180} \\
\% 0\end{array}$ & $\begin{array}{l}\delta \text { D } \\
\% 0\end{array}$ & $\begin{array}{c}\text { Precip. } \\
\text { Amt. } \\
\text { cm }\end{array}$ & Sample name & $\begin{array}{c}\delta^{18} 0 \\
\% 0\end{array}$ & $\begin{array}{l}\delta \mathbf{D} \\
\% 0\end{array}$ & $\begin{array}{c}\text { Precip. } \\
\text { Amt. } \\
\text { cm }\end{array}$ \\
\hline S. Portal 060418 & -4.6 & -43 & 0.25 & Site 1070425 & -9.6 & -75 & 0.23 & Site 1081103 & -8.5 & -70 & 0.44 \\
\hline Site 401060418 & -7.0 & -42 & 0.51 & Site 2070425 & -10.8 & -84 & 0.20 & Site 2081103 & -9.2 & -80 & 0.33 \\
\hline Site 418060418 & -4.9 & -39 & 0.28 & Site 401070425 & -11.4 & -89 & 0.15 & Site 401081103 & -10.3 & -79 & 0.33 \\
\hline Site 6060418 & -5.8 & -46 & 0.33 & Site 418070425 & -9.7 & -77 & 0.28 & Site 6081103 & -9.1 & -75 & 0.35 \\
\hline Site 8060418 & -5.3 & -45 & 0.23 & Site 6070425 & -11.7 & -97 & 0.38 & Site 8081103 & -8.6 & -73 & 0.44 \\
\hline S. Portal 060711 & -2.2 & -17 & 1.07 & Site 8070425 & -8.8 & -75 & 0.15 & S. Portal 081201 & -18.6 & -139 & 0.73 \\
\hline Site 1060711 & -2.3 & -17 & 0.97 & S. Portal 070806 & -4.0 & -38 & 0.13 & Site 1081201 & -18.9 & -137 & 0.75 \\
\hline Site 2060711 & -0.7 & -8 & 0.91 & Site 1070806 & -4.4 & -42 & 0.20 & Site 2081201 & -20.1 & -147 & 0.74 \\
\hline Site 401060711 & 0.0 & 1 & 0.38 & Site 2070806 & -4.3 & -40 & 0.20 & Site 401081201 & -20.4 & -147 & 0.73 \\
\hline Site 418060711 & 3.0 & 10 & 0.51 & Site 401070806 & -5.7 & -42 & 0.84 & Site 6081201 & -19.4 & -143 & 0.75 \\
\hline Site 6060711 & -2.3 & -18 & 0.41 & Site 418070807 & -2.7 & -31 & 0.13 & Site 8081201 & -18.6 & -134 & 0.80 \\
\hline Site 8060711 & -0.7 & -7 & 0.81 & Site 6070806 & -5.1 & -42 & 0.71 & S. Portal 081222 & -13.9 & -90 & 0.42 \\
\hline S. Portal 060722 & -2.4 & -32 & 1.35 & Site 8070806 & -3.3 & -33 & 0.10 & Site 1081222 & -13.6 & -93 & 0.44 \\
\hline Site 1060722 & -0.6 & -23 & 0.71 & S. Portal 070829 & -2.7 & -24 & 0.36 & Site 2081222 & -13.0 & -92 & 0.18 \\
\hline Site 2060722 & -1.1 & -24 & 0.79 & Site 1070829 & -1.3 & -19 & 0.28 & Site 401081222 & -12.6 & -85 & 0.28 \\
\hline Site 401060722 & -0.8 & -25 & 0.46 & Site 2070828 & -3.0 & -26 & 0.28 & Site 6081222 & -12.2 & -80 & 0.33 \\
\hline Site 418060722 & -1.7 & -29 & 1.60 & Site 401070828 & -5.1 & -38 & 0.43 & Site 8081222 & -13.9 & -97 & 0.46 \\
\hline Site 6060722 & -4.0 & -39 & 0.91 & Site 418070829 & -3.9 & -26 & 0.15 & S. Portal 081229 & -12.6 & -85 & 0.18 \\
\hline Site 8060722 & -3.2 & -33 & 1.42 & Site 6070829 & -1.1 & -19 & 0.20 & Site 1081229 & -12.5 & -91 & 0.19 \\
\hline S. Portal 060808 & -1.1 & -26 & 0.05 & Site 8070829 & -2.1 & -20 & 0.28 & Site 2081229 & -10.8 & -68 & 0.17 \\
\hline Site 2060808 & -2.3 & -30 & 0.15 & S. Portal 070925 & -12.5 & -82 & 7.37 & Site 401081229 & -10.8 & -67 & 0.23 \\
\hline Site 418060809 & 1.2 & -15 & 0.08 & Site 1070924 & -12.6 & -83 & 7.49 & Site 6081229 & -10.6 & -67 & 0.21 \\
\hline S. Portal 060912 & -2.8 & -38 & 0.30 & Site 2070924 & -13.0 & -85 & 7.39 & Site 8081229 & -12.4 & -83 & 0.16 \\
\hline Site 1060912 & -3.6 & -43 & 0.28 & Site 401070924 & -12.9 & -83 & 8.08 & S. Portal 090126 & -11.0 & -83 & 0.23 \\
\hline Site 2060911 & -7.2 & -55 & 0.30 & Site 418070925 & -12.5 & -84 & 6.81 & Site 1090126 & -10.7 & -77 & 0.21 \\
\hline Site 401060912 & -9.5 & -72 & 0.64 & Site 6070924 & -12.7 & -84 & 8.13 & Site 2090126 & -9.4 & -66 & 0.25 \\
\hline Site 418060911 & -5.5 & -45 & 0.25 & Site 8070924 & -12.4 & -86 & 7.37 & Site 401090126 & -9.3 & -63 & 0.28 \\
\hline Site 6060912 & -9.1 & -71 & 0.58 & S. Portal 071210 & -16.7 & -124 & 1.22 & Site 6090126 & -9.9 & -65 & 0.25 \\
\hline Site 8060912 & -2.9 & -38 & 0.20 & Site 1071210 & -16.7 & -129 & 1.14 & Site 8090126 & -10.8 & -88 & 0.22 \\
\hline S. Portal 061004 & -6.6 & -80 & 0.23 & Site 2071210 & -14.5 & -103 & 0.79 & & & & \\
\hline Site 1061003 & -7.2 & -81 & 0.18 & Site 401071210 & -11.9 & -85 & 0.33 & & & & \\
\hline
\end{tabular}




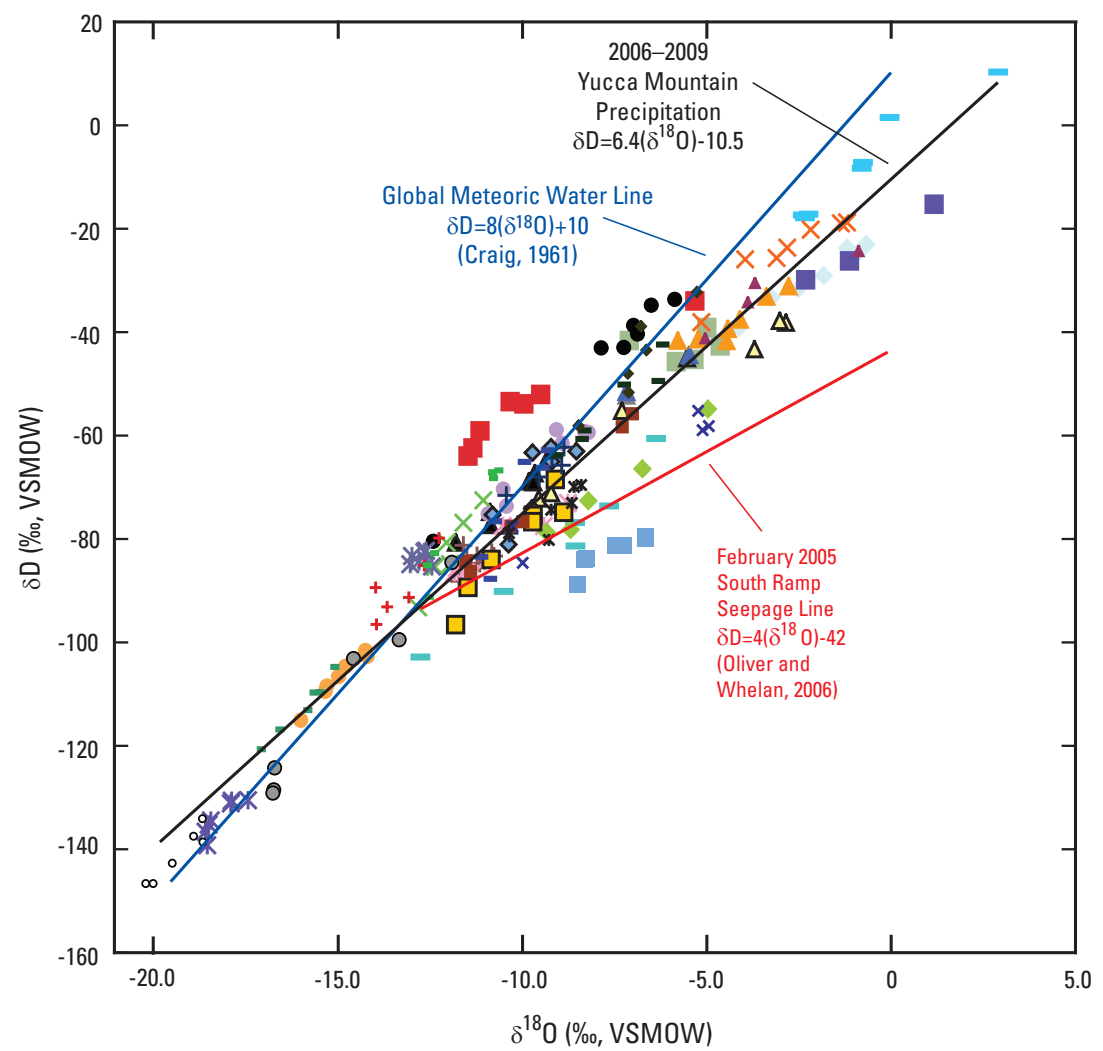

Precipitation event interval and collection date are expressed as month/day-day/year or month/day/year.

EXPLANATION

(Duration of precipitation event expressed as month/day-day/year or month/day/year)

\begin{tabular}{|c|c|c|c|}
\hline \multirow{2}{*}{$\begin{array}{r}\text { Event Interval } \\
2 / 18-19 / 06\end{array}$} & \multirow{2}{*}{$\begin{array}{c}\text { Collection Date } \\
2 / 21 / 06\end{array}$} & Event Interval & Collection Date \\
\hline & & $\square$ 4/22-23/07 & $4 / 25 / 07$ \\
\hline A $2 / 27-28 / 06$ & $3 / 1 / 06$ & $\Delta \quad 7 / 30-8 / 3 / 07$ & $8 / 6 / 07$ \\
\hline$\times 3 / 03-12 / 06$ & $3 / 13 / 06$ & $\times \quad 8 / 26-28 / 07$ & $8 / 29 / 07$ \\
\hline * 3/18-21/06 & $3 / 23 / 06$ & * 9/21-22/07 & $9 / 25 / 07$ \\
\hline - $3 / 28-29 / 06$ & $3 / 30 / 06$ & O 11/30-12/9/07 & $12 / 10 / 07$ \\
\hline$+4 / 3-6 / 06$ & $4 / 6 / 06$ & $+12 / 18-1 / 5 / 08$ & $1 / 8 / 08$ \\
\hline 4/10-17/06 & $4 / 18 / 06$ & - $1 / 24-27 / 08$ & $1 / 28 / 08$ \\
\hline$=7 / 8-10 / 06$ & $7 / 11 / 06$ & - $2 / 20-24 / 08$ & $2 / 25 / 08$ \\
\hline $7 / 20-22 / 06$ & $7 / 22 / 06$ & - 5/23-25/08 & $5 / 28 / 08$ \\
\hline 8/3/06 & $8 / 8 / 06$ & - 7/12-12/08 & $7 / 14 / 08$ \\
\hline$\Delta 9 / 6-7 / 06$ & $9 / 12 / 06$ & $\Delta \quad 9 / 8 / 08$ & $9 / 9 / 08$ \\
\hline $10 / 1-2 / 06$ & $10 / 4 / 06$ & $\times \quad 10 / 4 / 08$ & $10 / 6 / 08$ \\
\hline * 10/13-15/06 & $10 / 18 / 06$ & * 10/31-11/1/08 & $11 / 3 / 08$ \\
\hline - 12/9-10/06 & $12 / 11 / 06$ & $11 / 26 / 08$ & $12 / 1 / 08$ \\
\hline - $12 / 16-17 / 06$ & $12 / 18 / 06$ & $+12 / 15-17 / 08$ & $12 / 22 / 08$ \\
\hline$\Delta$ 2/11/07 & $2 / 14 / 07$ & $12 / 25-28 / 08$ & $12 / 29 / 08$ \\
\hline - 2/18-19/07 & $2 / 20 / 07$ & - $1 / 22-25 / 09$ & $1 / 26 / 09$ \\
\hline $3 / 20 / 07$ & $3 / 20 / 07$ & & \\
\hline$\diamond 4 / 15-16 / 07$ & $4 / 17 / 07$ & & \\
\hline
\end{tabular}

Figure 4. Delta deuterium plotted against delta oxygen-18, in per mil relative to Vienna Standard Mean Ocean Water, for 2006-2009 precipitation samples from monitoring stations at Yucca Mountain, Nevada. 


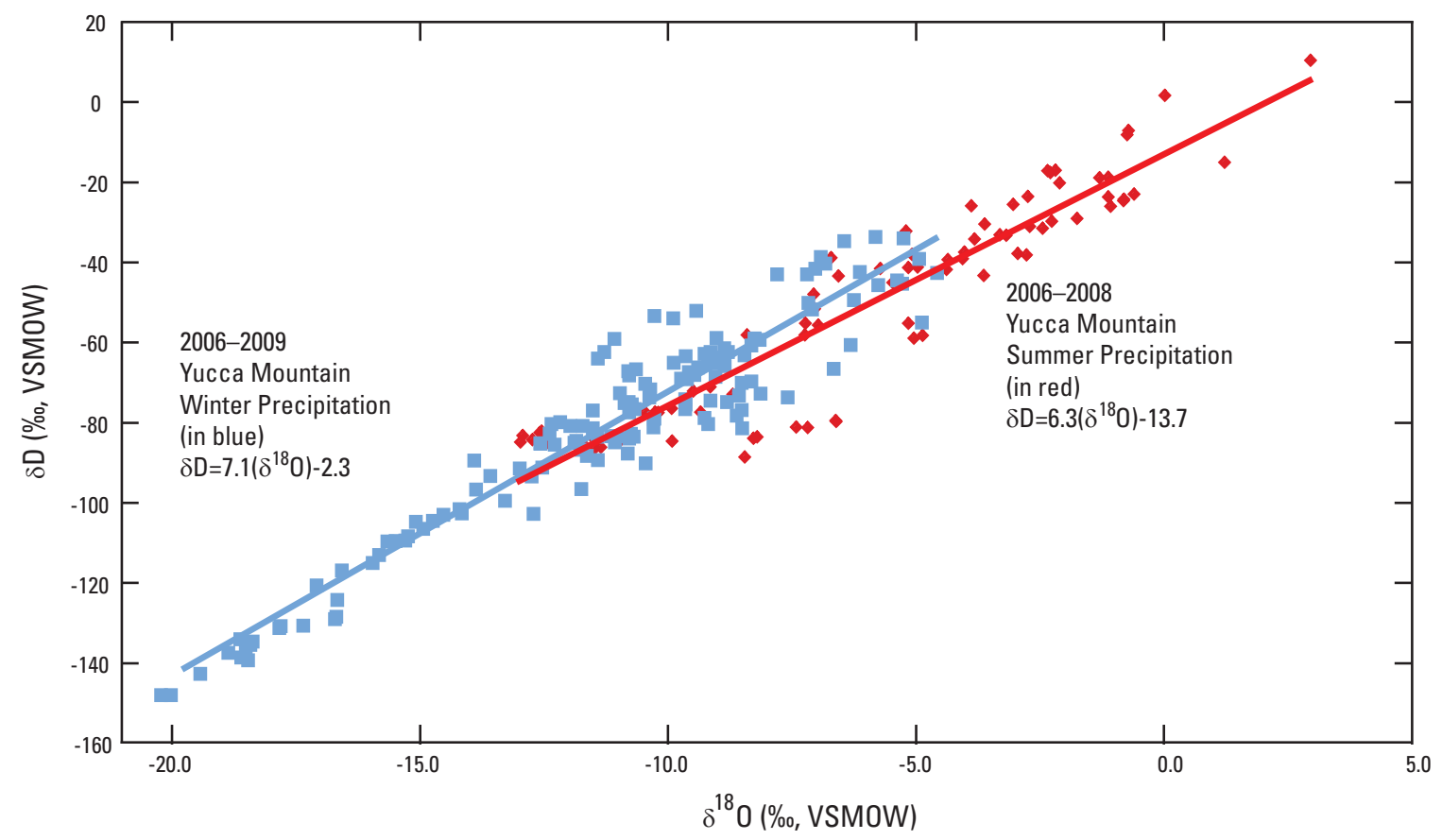

Figure 5. Delta deuterium plotted against delta oxygen-18, in per mil relative to Vienna Standard Mean Ocean Water, for 2006-2009 samples of winter (November-April) and summer (May-October) precipitation from monitoring stations at Yucca Mountain, Nevada.

The stable-isotopic composition of precipitation at and near Yucca Mountain has been measured intermittently over the past three decades by Ingraham and others (1990) for 1982-86, Milne and others (1987) and Benson and Klieforth (1989) for 1983-86, Whelan and others (1995) for 1993-95, and by Moscati and others (2008) for 2006-08. There also have been regional stable-isotopic studies of precipitation that encompassed southern Nevada by Friedman and others (1992; 2002) for 1982-89 and 1991-97. The stable-isotopic studies specific to the Yucca Mountain region yielded very similar results to data presented here; precipitation samples define an average line of slope of 6.7, and the seasonal range of $\delta^{18} \mathrm{O}$ and $\delta \mathrm{D}$ is consistent with the data presented in figure 4. Specifically, Whelan and others (1995) reported a summer line of slope of 5.8 and for winter, 7.8. In the current work (fig. 5), the summer and winter lines of slopes are 6.3 and 7.1, respectively.

In general, the two data sets are complementary; however, the Whelan and others (1995) data locally contained incomplete data sets and analyses from multiple precipitation events (in some cases spanning over 8 weeks), due to locations inaccessible during winter and (or) due to a lack of resources to collect samples. Data in this report (table 3, fig. 4) also compare favorably with the precipitation data collected during the mid-1980s by Benson and Klieforth (1989). Although Benson and Klieforth did not specifically compare summer and winter precipitation, they did compare precipitation that fell only as rain to that which fell only as snow. Their rain-only data defined a line of slope of 6.6 , and their snow-only data defined a line of 8 (identical to the GMWL because snow does not fractionate due to evaporation). A compilation of 342 pairs $\left(\delta^{18} \mathrm{O}\right.$ and $\left.\delta \mathrm{D}\right)$ of precipitation data collected by Whelan and others (1995) in the early 1990s with the data reported here provide an annual mean value of -10.3 and $-76 \%$ o $\left(\delta^{18} \mathrm{O}\right.$ and $\delta \mathrm{D}$, respectively) for Yucca Mountain precipitation.

Seasonal isotopic variation principally is a result of temperature differences but also is affected by seasonal changes in moisture sources and storm-track trajectories. Friedman and others (1992) analyzed storm tracks and demonstrated that winter storms in the Yucca Mountain area typically originate from the Pacific Ocean and pass over high mountain ranges in California before reaching the Yucca Mountain area. The heavy isotopes in water, ${ }^{18} \mathrm{O}$ and $\mathrm{D}$, are fractionated preferentially into the liquid phase during condensation. Therefore, precipitation (or rain-out) from moist, cooling air masses during adiabatic uplift over high terrain decreases $\delta^{18} \mathrm{O}$ and $\delta \mathrm{D}$ values of remaining water vapor. In contrast, summer storms typically arrive from the south or east, obtaining 
water vapor from the Gulf of California or the Gulf Mexico, without traversing substantial mountainous terrain. Hence, typical summer storm trajectories allow the air masses to reach Yucca Mountain with less fractionation of initial isotopic composition(s) and consequently have isotopic values that are higher than their winter counterparts. Winter precipitation, in the form of rain, undergoes only minor evaporation as it falls from cloud to ground. If winter precipitation falls as snow, the snow may sublimate as it falls, but without fractionation of the isotopic composition. As such, winter samples, in general, plot closer to, or even above, the GMWL (fig. 4), in contrast to summer precipitation, which is subject to more evaporation due to warmer air temperatures.

\section{Conclusions}

Monitoring of modern precipitation for stable-isotope and chemical compositions provides baseline hydrochemical data of Yucca Mountain precipitation, the major source of infiltration to the unsaturated zone and of recharge to the saturated zone. Those baseline data constitute an invaluable component for many types of studies, including characterization of potential seepage into the tunnels of a formerly proposed repository; groundwater origins, recharge, and movement through the unsaturated and saturated zones; infiltration and evaporation rates; and water/rock interaction.

Chemical analyses of the precipitation indicate that the samples are dilute, perhaps a function of the length of the collection period (typically spanning several days of rainfall). The precipitation has $\mathrm{pH}$ values of 6-7, slightly less acidic than most precipitation, likely due to buffering by local naturally alkaline dust that contains a large fraction of calcium carbonate. The cation composition of the precipitation generally is dominated by calcium, while the anion composition is marked by high sulfate and nitrate.

Stable isotopic and chemical analysis of samples of Yucca Mountain precipitation in 2006-09 has yielded a snapshot of baseline hydrochemical inputs of meteoric water that will provide greater understanding of potential future seepage events in the area. Over the 3 years of this study, sampled $\delta^{18} \mathrm{O}$ and $\delta \mathrm{D}$ values ranged from 3.0 to $-20.4 \%$ and 10 to $-147 \%$, respectively. The $\delta^{18} \mathrm{O}$ and $\delta \mathrm{D}$ values plot on a line of slope of 6.4 (less than the slope of 8 of the GMWL), a result of evaporation of falling raindrops typical of arid environments. Winter-summer seasonal variation is readily apparent with average winter precipitation values of -11.9 and $-81 \%$ o and average summer precipitation values of -5.9 and $-51 \%$ o $\left(\delta^{18} \mathrm{O}\right.$ and $\delta \mathrm{D}$, respectively). Although seasonal shifts principally are due to temperature, changes in moisture sources and storm track trajectories also play a role. A cumulative analysis of 342 available pairs $\left(\delta^{18} \mathrm{O}\right.$ and $\left.\delta \mathrm{D}\right)$ of stable isotopic data generated by the U.S. Geological Survey from the early 1990s to the present (this work) yields an annual mean value for $\delta^{18} \mathrm{O}$ of $-10.3 \%$ and for $\delta \mathrm{D}$ of $-76 \%$ for Yucca Mountain precipitation.

\section{Acknowledgments}

We would like to thank Paul Fransioli, Tim Moran, Gary Jones (Bechtel SAIC Company), and Shawn Otto (Los Alamos National Laboratory) for their help installing the collection network and tirelessly collecting and shipping samples after each precipitation event. Cayce Gulbransen (US Geological Survey) assisted with mass spectrometry analyses and Tom Oliver (SM Stoller Corporation) assisted with ion chromatography advice. Andy Hunt, Pete Striffler, and Pamela Daddow (US Geological Survey) are thanked for providing constructive and thoughtful reviews of this report.

\section{References Cited}

Back, W., 1966, Hydrochemical facies and ground-water flow patterns in northern part of Atlantic Coastal Plain: U.S. Geological Survey Professional Paper 498-A, 42 p.

Bensen, L.V., and Klieforth, H., 1989, Stable isotopes in precipitation and ground water in the Yucca Mountain region, Southern Nevada-Paleoclimatic implications, in Peterson, D.H., ed., Aspects of climate variability in the Pacific and Western Americas: Washington, D.C., American Geophysical Union, Geophysical Monograph 55, p. 41-59.

Buesch, D.C., Spengler, R.W., Moyer, T.C., and Geslin, J.K., 1996, Proposed stratigraphic nomenclature and macroscopic identification of lithostratigraphic units of the Paintbrush Group exposed at Yucca Mountain, Nevada: U.S. Geological Survey Open-File Report 94-469, 47 p.

Claassen, H.C., and Halm, D.R., 1994, Performance characteristics of a bulk precipitation collector with respect to measurement of water isotopes oxygen-18 and deuterium: U.S. Geological Survey Water-Resources Investigations Report 94-4008, 3 p.

Craig, H., 1961, Isotopic variations in natural waters: Science, v. 133 , p. $1702-1703$.

Drever, J.I., 1997, The geochemistry of natural waters - Surface and groundwater environments: Englewood Cliffs, N.J., Prentice Hall, Inc., 3d ed., 436 p.

Flint, A.L., Flint, L.E., Kwicklis, E.M., Fabryka-Martin, J.T., and Bodvarsson, G.S., 2002, Estimating recharge at Yucca Mountain, Nevada, USA - Comparison of methods: Hydrogeology Journal, v. 10, p. 180-204, doi 10.1007/s10040001-0169-1. 
Fransioli, P., 2007, Local meteorology of Yucca Mountain, Nevada, 1994-2006. Prepared for the U.S. Department of Energy Yucca Mountain Project by Bechtel SAIC Company, LLC, Las Vegas, Nevada, 326 p. Accessed June 9, 2008, at http://www.lsnnet.gov [search under Participant Accession Number DOC.20070905.0008].

Friedman, I., Smith, G.I., Gleason, J.D., Warden, A., and Harris, J.M., 1992, Stable isotope composition of waters in southeastern California-1. Modern precipitation: Journal of Geophysical Research, v. 97, no. D5, p. 5795-5812.

Friedman, I., Smith, G.I., Johnson, C.A., and Moscati, R.J., 2002, Stable isotope compositions of waters in the Great Basin, United States-2. Modern precipitation: Journal of Geophysical Research, v. 107, no. D19, 4,401, p. 15-1 15-22, doi:10.1029/2001JD000566.

Ingraham, N.L., Jacobson, R.L., Hess, J.W., and Lyles, B.F., 1990, Stable isotopic study of precipitation and spring discharge on the Nevada Test Site: Desert Research Institute, University of Nevada, Publication 45078, 55 p.

Kendall, C., and Coplen, T.B., 1985, Multisample conversion of water to hydrogen by zinc for stable isotope determination: Analytical Chemistry, v. 57, p. 1437-1440.

Levich, R.A., and Stuckless, J.S., 2007, Yucca Mountain, Nevada-A proposed geologic repository for high-level radioactive waste, in Stuckless, J.S., and Levich, R.A., eds., The geology and climatology of Yucca Mountain and vicinity, southern Nevada and California: Geological Society of America Memoir 199, p. 1-7, doi:10.1130/2007.1199(05).

Milne, W.K., Benson, L.V., and McKinley, P.W., 1987, Isotope content and temperature of precipitation in southern Nevada, August 1983-August 1986: U.S. Geological Survey Open-File Report 87-463, 37 p.

Moscati, R.J., Scofield, K.M., Fransioli, P.M., Moran, T.M., and Jones, G.W., 2008, Isotope and chemical monitoring of modern precipitation, Yucca Mountain, Nevada, in HighLevel Radioactive Waste Management, Proceedings of the 12th International Conference, Las Vegas, Nevada: La Grange Park, Illinois, American Nuclear Society, p. 204-213.

O’Dell, J.W., Pfaff, J.D., Gales, M.E., and McKee, G.D., 1984, The determination of inorganic ions in water by ion chromatography-EPA Test Method 300.0: U.S. Environmental Protection Agency, Environmental Monitoring Systems Laboratory, Cincinnati, Ohio, Report No. EPA/600/4$84 / 017,5$ p.
Oliver, T., and Whelan, J., 2006, Characterization of seepage in the Exploratory Studies Facility, Yucca Mountain, Nevada, in High-Level Radioactive Waste Management, Proceedings of the 11th International Conference, Las Vegas, Nevada: La Grange Park, Illinois, American Nuclear Society, p. 357-366.

Reheis, M.C., and Kihl, R., 1995, Dust deposition in southern Nevada and California, 1984-1989-Relations to climate, source area, and source lithology: Journal of Geophysical Research, v. 100, no. D5, p. 8893-8918.

Sawyer, D.A., Fleck, R.J., Lanphere, M.A., Warren, R.G., Broxton, D.E., and Hudson, M.R., 1994, Episodic caldera volcanism in the Miocene southwestern Nevada volcanic field-Revised stratigraphic framework, 40Ar/39Ar geochronology, and implications for magmatism and extension: Geological Society of America Bulletin, v. 106, no. 10, p. 1304-1318.

Sharpe, S.E., 2007, Using modern through mid-Pleistocene climate proxy data to bound future variations in infiltration at Yucca Mountain, Nevada, in Stuckless, J.S., and Levich, R.A., eds., The geology and climatology of Yucca Mountain and vicinity, southern Nevada and California: Geological Society of America Memoir 199, p. 155-205, doi:10.1130/2007.1199(05).

Whelan, J.F., Moscati, R.J., and Allerton, S.B.M., 1995, Stable isotopic composition of precipitation at Yucca Mountain and the surrounding region-Feb. 1993 to Feb. 1995: Milestone Report to the U.S. Department of Energy, Yucca Mountain Project. Accessed June 9, 2008, at http://www.lsnnet. gov [search under Participant Accession Number MOL. 19960220.0022].

Publishing support provided by: Denver Publishing Service Center

For more information concerning this publication, contact: Office of Groundwater Headquarters

Box 25046, Mail Stop 963

Denver, CO 80225

Or visit the Colorado Water Science Center Web site at: http://co.water.usgs.gov/ 